\title{
PD-L1 signaling in reactive astrocytes counteracts neuroinflammation and ameliorates neuronal damage after traumatic brain injury
}

\author{
Xiang Gao ${ }^{1^{*}}$, Wei Li ${ }^{2 \dagger}$, Fahim Syed ${ }^{2}$, Fang Yuan ${ }^{1}$, Ping $\mathrm{Li}^{3}$ and Qigui Yu ${ }^{2 *}$
}

\begin{abstract}
Background: Tissue damage and cellular destruction are the major events in traumatic brain injury (TBI), which trigger sterile neuroimmune and neuroinflammatory responses in the brain. While appropriate acute and transient neuroimmune and neuroinflammatory responses facilitate the repair and adaptation of injured brain tissues, prolonged and excessive neuroimmune and neuroinflammatory responses exacerbate brain damage. The mechanisms that control the intensity and duration of neuroimmune and neuroinflammatory responses in TBI largely remain elusive.

Methods: We used the controlled cortical impact (CCI) model of TBI to study the role of immune checkpoints (ICPs), key regulators of immune homeostasis, in the regulation of neuroimmune and neuroinflammatory responses in the brain in vivo.

Results: We found that de novo expression of PD-L1, a potent inhibitory ICP, was robustly and transiently induced in reactive astrocytes, but not in microglia, neurons, or oligodendrocyte progenitor cells (OPCS). These PD-L1+ reactive astrocytes were highly enriched to form a dense zone around the TBI lesion. Blockade of PD-L1 signaling enlarged brain tissue cavity size, increased infiltration of inflammatory Ly- $6 C^{\text {High }}$ monocytes/macrophages (M/M $\phi$ ) but not tissue-repairing Ly-6C $\mathrm{CW}_{\mathrm{F}} 4 / 80^{+} \mathrm{M} / \mathrm{M} \phi$, and worsened TBI outcomes in mice. PD-L1 gene knockout enhanced production of CCL2 that is best known for its ability to interact with its cognate receptor CCR2 on Ly- $6 C^{\text {High }} \mathrm{M} / \mathrm{M} \varphi$ to chemotactically recruit these cells into inflammatory sites. Mechanically, PD-L1 signaling in astrocytes likely exhibits dual inhibitory activities for the prevention of excessive neuroimmune and neuroinflammatory responses to TBI through (1) the PD-1/PD-L1 axis to suppress the activity of brain-infiltrating PD- $1^{+}$immune cells, such as PD- $1^{+}$T cells, and (2) PD-L1 intrinsic signaling to regulate the timing and intensity of astrocyte reactions to TBI.
\end{abstract}

Conclusions: PD-L1+ astrocytes act as a gatekeeper to the brain to control TBI-related neuroimmune and neuroinflammatory responses, thereby opening a novel avenue to study the role of ICP-neuroimmune axes in the pathophysiology of TBI and other neurological disorders.

\footnotetext{
*Correspondence: xiangao@iupui.edu; andyu@iupui.edu

${ }^{\dagger}$ Xiang Gao and Wei Li contributed equally to this work

${ }^{1}$ Spinal Cord and Brain Injury Research Group, Department

of Neurological Surgery, Stark Neurosciences Research Institute, Indiana

University School of Medicine, 320 W. 15th Street, Indianapolis, IN 46202,

USA

2 Department of Microbiology and Immunology, Medical Science

Building, MS267, Indiana University School of Medicine, 635 Barnhill Drive,

Indianapolis, IN 46202, USA

Full list of author information is available at the end of the article
}

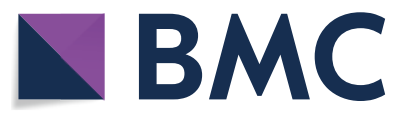

(c) The Author(s) 2022. Open Access This article is licensed under a Creative Commons Attribution 4.0 International License, which permits use, sharing, adaptation, distribution and reproduction in any medium or format, as long as you give appropriate credit to the original author(s) and the source, provide a link to the Creative Commons licence, and indicate if changes were made. The images or other third party material in this article are included in the article's Creative Commons licence, unless indicated otherwise in a credit line to the material. If material is not included in the article's Creative Commons licence and your intended use is not permitted by statutory regulation or exceeds the permitted use, you will need to obtain permission directly from the copyright holder. To view a copy of this licence, visit http://creativecommons.org/licenses/by/4.0/. The Creative Commons Public Domain Dedication waiver (http://creativeco mmons.org/publicdomain/zero/1.0/) applies to the data made available in this article, unless otherwise stated in a credit line to the data. 
Keywords: Traumatic brain injury, Astrocyte, Immune checkpoint, PD-L1, Intrinsic signaling, Neuroimmune response, Neuroinflammation

\section{Background}

Traumatic brain injury (TBI) is a major cause of death and disability worldwide, affecting more than 10 million people annually [1-3]. TBI is composed of primary and secondary injuries. Primary injury refers to the initial direct impact that causes focal and diffuse damage of the brain. Other than injury prevention, little can be done to interfere with the primary injury [4]. The secondary injury of TBI develops in minutes to hours following the initial primary injury and contributes to further damage of brain tissue $[3,5,6]$. In fact, secondary injury remains an important determinant of TBI outcome, as a large percentage of people with TBI develop neurological function deficits days to weeks after the event. Secondary injury is an indirect result of destructive immunological, inflammatory, neurotoxic, and biochemical cascades that are initially triggered by the primary injury. However, the exact roles of the neuroimmune and neuroinflammatory responses in the physiological and pathological processes of secondary injury are largely unknown.

Tissue damage and cellular destruction are the major events in TBI primary injury, which trigger sterile neuroimmune and neuroinflammatory responses in the absence of microbial infection [7]. Neuroimmune and neuroinflammatory responses in TBI are characterized by glial cell activation, leukocyte recruitment, and upregulation of inflammatory mediators $[8,9]$, and can have both beneficial and detrimental effects [9-11]. TBI damages the blood-brain barrier (BBB), enabling recruitment of circulating immune cells, such as monocytes/ macrophages $(\mathrm{M} / \mathrm{M} \phi)$, neutrophils, and activated T cells to the injured site. The accumulation of blood-borne immune cells is found in the brain parenchyma of human TBI and animal models of TBI [10]. These infiltrated cells release inflammatory mediators that mobilize immune cells and neural cells, such as glia to the site of injury [10]. Resident neural cells such as astrocytes and microglia are also activated and subsequently form an area of containment between injured and healthy tissues, suggesting that acute activation of resident neural cells may represent the first line of defense following TBI $[10,12]$. However, when resident neural cells and infiltrated immune cells become over-activated, they can induce detrimental neurotoxic effects by releasing cytotoxic substances, such as pro-inflammatory cytokines (IFN- $\gamma$, IL- $1 \beta$, and TNF$\alpha)[10,13]$. These inflammatory cytokines can further activate neural cells such as astrocytes to significantly affect the pathophysiology of TBI [14]. Thus, the intensity and duration of neuroimmune and neuroinflammatory responses are critical for determining their supportive or destructive effects on the central nervous system (CNS) post-TBI.

The immune responses in the brain parenchyma are tightly regulated to prevent overwhelming immune activation and subsequent inflammatory responses in this organ that has little recovery capacity $[15,16]$. Since immune checkpoints (ICPs) are key regulatory molecules that maintain immune homeostasis, they likely play a critical role in regulating neuroimmune and neuroinflammatory responses in TBI. Most ICPs consist of paired receptor-ligand molecules that exert inhibitory or stimulatory effects on immune defense, surveillance, regulation, and self-tolerance [17-21]. Under normal circumstances, ICPs regulate the breadth, magnitude, and duration of the immune responses against malignancy and infection while protecting tissues from excessive insults. In certain pathological situations such as cancer or persistent infection, the balance between ICP stimulatory and inhibitory signals is dysregulated [17-25]. Malignant cells or persistent infections can dysregulate the expression of ICPs, such as CTLA-4 (cytotoxic T-lymphocyte-associated protein 4), PD-1 (programmed cell death protein 1), PD-L1 (programmed death-ligand 1), TIM-3 (T-cell immunoglobulin mucin protein 3 ), and LAG-3 (lymphocyte-activation gene 3 ) on the surface of immune cells to evade or subvert the immune response, leading to insufficiency or failure in anti-tumor or pathogen immune attacks [22, 26-28]. These important findings have laid the foundation for the clinical development of ICP blockade therapies, which abrogate ICP inhibitory signals to restore and enhance the anti-tumor activity of cytotoxic T lymphocytes (CTLs) [19, 20, 29, 30]. In fact, ICP blockade has become a revolutionary treatment for several advanced malignancies, such as melanoma and lung cancer [15, 20,31].

Currently, there has been little research conducted to study the biology and clinical relevance of ICPs in the brain after TBI. We used the controlled cortical impact (CCI) model of TBI to study the role of ICPs in the regulation of neuroimmune and neuroinflammatory responses in the brain. We found that de novo expression of PD-L1, a key inhibitory ICP that exerts dual inhibitory signals to suppress the activity of $\mathrm{PD}-1^{+} \mathrm{T}$ cells via the PD-1/PD-L1 axis and the function of PD-L1 ${ }^{+}$cells via a novel intrinsic signaling, was robustly and specifically induced in astrocytes that were positive for GFAP (glial 
fibrillary acidic protein), a reactive astrocyte marker. These PD-L1 ${ }^{+}$astrocytes were highly enriched to form a dense zone around the TBI lesion. Blockade of PD-L1 signaling increased infiltration of inflammatory Ly- $6 \mathrm{C}^{\text {High }}$ $\mathrm{M} / \mathrm{M} \phi$, brain tissue cavity size, and motor and emotional dysfunction of TBI mice. Therefore, our study reveals that $\mathrm{PD}-\mathrm{L}^{+}$reactive astrocytes act as a gatekeeper to control TBI-related neuroimmune and neuroinflammatory responses, thereby opening a novel avenue to investigate the role of ICP-neuroimmune axes in TBI pathophysiology. In addition, our results provide insights into the development of ICP regulators to improve TBI clinical outcomes.

\section{Materials and methods \\ Animals}

Studies have shown that men have a significantly higher incidence of TBI than women, particularly during young adulthood [32]. In addition, TBI-related hospitalization and death in men are much higher than that in women [33]. Therefore, this study focuses on using male mice as TBI research subjects. Male C57BL/6 mice at 8-12 weeks of age (Jackson Laboratories, Bar Harbor, ME) were used in all experiments. They were housed in an animal facility with a 12/12 light/dark cycle, were supplied with a standard pellet diet, and had free access to water ad libitum. All animal procedures were approved by Indiana University Institutional Animal Care and Use Committee (IACUC).

\section{Controlled cortical impact model of traumatic brain injury (CCI-TBI)}

Mice (8-12 weeks of age) were subjected to moderate CCI-TBI or sham using sterile procedures as we previously described [34-36]. Briefly, the mice were anesthetized using a solution of 2.5\% Avertin (Sigma-Aldrich, St Louis, MO) via intraperitoneal (IP) injection. The mouse head was fixed in the stereotaxic frame (Kopf Instruments, Tujunga, CA) using the ear bars and bite plate. A 4-mm diameter craniotomy with a midway between the bregma and lambda sutures and laterally halfway between the central suture and the temporalis muscle was performed to remove the skullcap without disruption of the underlying dura. Prior to performing CCI-TBI, the tip of the electromagnetic impactor was adjusted and kept perpendicular to the exposed cortical surface. CCI injuries were produced using an electromagnetic impactor (Impact One, Leica Biosystems, Buffalo Grove, IL). The piston velocity was set at $3.5 \mathrm{~m} / \mathrm{s}$, depth of penetration of $1.0 \mathrm{~mm}$, and dwell time of $100 \mathrm{~ms}$. This CCI setting resulted in an injury of moderate severity. After CCI-TBI, the cranial exposure was closed using sutures. Sham mice had a craniotomy without CCI impact. During surgery and recovery, the core body temperature of the animals was maintained at $36-37^{\circ} \mathrm{C}$ using a heating pad. All animals survived the injury and subsequent experiments.

\section{Administration of PD-L1 blocking or tracking antibody}

TBI damages the BBB, enabling recruitment of circulating immune cells, such as $\mathrm{M} / \mathrm{M} \phi$, neutrophils, and activated $\mathrm{T}$ cells to the injured site. We also took advantage of the injury-induced transient breakdown of the BBB to study whether administration of PD-L1 blocking antibody $(\mathrm{Ab})$ via subcutaneous $(S C)$ injection could reach the injured site of the brain to exert its blocking function. Previous studies have shown that a single dose of anti-PD-L1 Ab $(50 \mu \mathrm{g} / \mathrm{kg}$ or $200 \mu \mathrm{g} / \mathrm{kg})$ via intravenous or intraperitoneal injection can significantly affect stroke outcome through regulating CNS inflammation [37, 38]. In our study, mice at $24 \mathrm{~h}$ post-TBI were subcutaneously injected with either PD-L1 blocking Ab (ab233482, Abcam, Cambridge, MA), PD-L1-Alex 647-conjugated Ab (ab224030, Abcam, Cambridge, MA), or an irrelevant IgG $\mathrm{Ab}$ as a control at $200 \mu \mathrm{g} / \mathrm{kg}$ in $100 \mu \mathrm{L}$ PBS. PD-L1-Alex 647-conjugated $\mathrm{Ab}$ was used for histological examination of $\mathrm{Ab}$ penetration into the brain, while PD-L1 blocking Ab was used to study the PD-L1 function in the brain. The treatments were blinded to researchers who performed histological evaluations, behavior tests, and flow cytometry.

\section{Tissue processing and Nissl staining}

Tissue processing and Nissl staining were performed as described in our previous report [36]. Briefly, anesthetized mice were intracardially perfused with heparinized PBS to remove blood, followed by fixation with $4 \%$ paraformaldehyde (PFA) in PBS. The brain tissues were collected and post-fixed overnight with PFA in $4{ }^{\circ} \mathrm{C}$, followed by cryoprotection in $30 \%$ sucrose for $48 \mathrm{~h}$. Serial coronal sections were cut at $30 \mu \mathrm{m}$ using a cryostat (LeicaCM 1950; Leica, Buffalo Grove, IL) and preserved at $-20{ }^{\circ} \mathrm{C}$. Nissl staining was used to analyze histological changes of the brain, including lesion cavity measurement. Briefly, sections were incubated in a solution of $0.1 \%$ cresyl violet (Sigma, St Louis, MO) for 20 min. After a quick rinse in distilled water, the sections were differentiated in 95\% ethanol for $3 \mathrm{~min}$, followed by dehydration in $100 \%$ ethanol for $2 \times 5 \mathrm{~min}$. The sections were cleared in xylene for $2 \times 5 \mathrm{~min}$, air-dried, and mounted with DPX Mountant (Sigma-Aldrich, St Louis, MO). Slides were viewed using an upright microscopy system that was interfaced with a computer-controlled digital camera (Zeiss Axio Imager M2, Oberkochen, Germany). Images were captured and processed using AxioVision v4.8 software (Oberkochen, Germany). 


\section{Immunohistochemistry (IHC) analysis}

Brain tissue sections were incubated in blocking solution (0.1\% Triton $\mathrm{X}-100,1 \%$ bovine serum albumin, and $5 \%$ normal serum in PBS) for $1 \mathrm{~h}$ at room temperature, followed by overnight incubation at $4{ }^{\circ} \mathrm{C}$ with primary antibodies (Abs) against mouse PD-L1, GFAP, Iba1 (ionized calcium binding adaptor molecule 1), NeuN (neuronal nuclei), NG2 (nerve/glial antigen 2), and CD45. The sections were washed 3 times with PBS, and incubated at room temperature for $2 \mathrm{~h}$ with fluorochrome-conjugated secondary Abs. After staining of nuclear DNA with DAPI (4',6-diamidino-2-phenylindole) for $2 \mathrm{~min}$, the sections were washed 3 times with PBS and were mounted on the slides using Fluorescent-G mounting medium (Invitrogen, Carlsbad, CA). After air drying, slides were covered using Fluorescent-G mounting medium (Invitrogen, Carlsbad, CA) for fluorescence preservation and enhancing. Final concentrations of primary Abs were used as follows: anti-PD-L1 (1:200, ab233482, Abcam, Cambridge, MA), PD-L1-Alex 647-conjugated Ab (ab224030, Abcam, Cambridge, MA), anti-GFAP (1:1000, G3893, Sigma-Aldrich, St. Louis, MO), anti-Iba1 (1:200, ab5076, Abcam, Cambridge, MA), anti-NeuN (1:1000, MAB377, Millipore, Billerica, MA), anti-NG2 (1:200, ab50009, Abcam, Cambridge, MA), anti-CD45 (1:200, ab10558, Abcam, Cambridge, MA), or an isotype $\mathrm{Ab}$ as a control staining. Secondary Abs (Jackson ImmunoResearch Laboratories, West Grove, PA) were applied in a dilution of $1: 1000$.

\section{Fluorescent intensity measurement and cell counting}

The intensity of PD-L1 or GFAP signal was measured using ImageJ software (version 1.47; NIH, Bethesda, MD). Briefly, images with PD-L1-positive signal or GFAP signal were captured using a Zeiss inverted microscope (Zeiss Axio 200, Oberkochen, Germany). The contour of injured cortex (epicenter) or scar formation area (within $400 \mu \mathrm{m}$ to the edge of cavity) was drawn using ImageJ software and the total PD-L1-positive signal or mean intensity of GFAP signal within the contour was measured. The average of total or mean intensity from five mice was used for comparing PD-L1-positive signals in the injured cortex among the different groups or GFAP signals between IgG control and PD-L1 Ab treatment TBI groups, respectively.

The PD-L1 and GFAP double positive reactive astrocytes were counted as described in our previous report [39]. The contours of 3 measuring areas (the block of injured cortex with $200 \mu \mathrm{m}$ wide from different distances to the edge of the cavity) were drawn. The GFAP-positive cells were quantified using an optical fractionator (MBF Bioscience, Williston, VT), while PD-L1/GFAP double positive $\left(\mathrm{PD}-\mathrm{L}^{+} / \mathrm{GFAP}^{+}\right)$cells were determined using a PD-L1 signal as an indicator. When a PD-L1 signal showed up, the channel was switched to look for a GFAP signal. If a GFAP signal was also detected, these cells were counted as PD-L1 $1^{+} / \mathrm{GFAP}^{+}$cells. The PD-L1 ${ }^{+} /$ $\mathrm{GFAP}^{+}$reactive astrocytes were expressed as percentage in total $\mathrm{GFAP}^{+}$reactive astrocytes, and the data from five mice was averaged and presented.

\section{Cortex cavity measurement}

The cavity volume was calculated as described previously [40]. Briefly, a series of every sixth sections $(30 \mu \mathrm{m}$ thickness, $180 \mu \mathrm{m}$ apart) covering the injured cortex were subjected to Nissl staining (cresyl violet) to show the spared cortex. The boundary contours of the contralateral and ipsilateral spared cortex were drawn with a Zeiss AxioVision v4.8 software (Oberkochen, Germany). The contours' enclosed volumes were measured. The percent cortex of the cavity was calculated with the following formula: percentage of the cortical cavity $=($ contralateral cortex volume-ipsilateral spare cortex volume)/ contralateral cortex volume $\times 100 \%$. Four groups of mice: sham + IgG, sham + PD-L1 Ab, TBI + IgG, and $\mathrm{TBI}+\mathrm{PD}-\mathrm{L} 1 \mathrm{Ab}$ were included with 5 mice in each group.

\section{Rotarod and elevated plus maze (EPM) tests}

Motor deficit and impulsivity are common manifests after TBI [41, 42], which can be commonly evaluated using the rotarod test or EPM test, respectively. The rotarod test was conducted first to evaluate the effects of anti-PD-L1 Ab treatment on balance and coordination of mice post-TBI versus sham as described in our previous report [43]. The rotating rod was $3 \mathrm{~cm}$ in diameter and divided by flanges in five compartments to allow testing of up to five mice simultaneously. The animals had to walk on the rotating rod from the lowest speed to gradually increased speed (0-30 rpm within $90 \mathrm{~s})$ and the time until the mouse fell from the rod (latency to fall) was recorded. Mice were rested for 20 min between every two tests. A total of 4 trials were performed for each timepoint.

Following the Rotarod test, the EPM test was performed to assess anxiety-related behavior of mice as previously described [44]. The EPM apparatus consists of a cross-shape platform with two oppositely positioned closed arms with high walls, two oppositely positioned open arms, and a small center area. The EPM apparatus was placed at $0.5 \mathrm{~m}$ from the ground. The EPM test is based on the natural aversion of rodents to open spaces. Mice were allowed to start at the maze center facing one of the open arms to explore the maze for a specific amount of time. As mice freely explored the maze, their 
behavior was recorded by a video camera mounted above the maze. Mouse behavioral metrics including total distance traveled and time spent in each arm were analyzed using ANY-maze software (Stoelting, Wood Dale, IL). The preference for being in open arms over closed arms (expressed as moving distance and time spent in the open arms) was calculated to measure anxiety-related behavior.

Four groups with 10 mice for each were used for behavior tests including: sham + IgG, sham + PD-L1 Ab, $\mathrm{TBI}+\mathrm{IgG}$, and TBI + PD-L1 Ab.

\section{Collection of cerebrospinal fluid (CSF), blood, brain, and spleen}

CSF was collected from mice with TBI or sham as previously reported [45]. Briefly, anesthetized mice were shaved at the back of the head above the eyes between the ears to the bottom of the neck using a razor blade for hair removal. The head of the mouse was secured using a mouse adapter. The skin and muscle were cut until the base of the skull was exposed. To collect CSF, a sharpened glass capillary (inner diameter $0.75 \mathrm{~mm}$, outer diameter $1.0 \mathrm{~mm}$ ) was placed into the capillary holder that was connected to a collecting syringe and firmly mounted on a micromanipulator. The glass capillary tip (inner diameter: $10-20 \mu \mathrm{m}$ ) was aligned to the back of the mouse head so that the sharpened point was just behind the membrane at a 30-45-degree angle. The micromanipulator was used to move the capillary tip closer to the membrane until resistance occurred. The capillary tube was gently tapped through the membrane by knocking on the micromanipulator controls under microscope monitoring. CSF was automatically drawn into the capillary tube once an opening was punctured. Approximately 5-10 $\mu \mathrm{l}$ of CSF per mouse was collected. After collection, CSF was centrifuged for $10 \mathrm{~s}$ at maximal speed using a mini centrifuge, and the supernatant was stored at $-80{ }^{\circ} \mathrm{C}$ until use. The heating pad was used to maintain the core body temperature at $36-37^{\circ} \mathrm{C}$ during CSF collection.

After the collection of CSF, mice were used for collecting blood, spleen, and brain. Blood was collected through direct cardiac blood withdrawal without an anticoagulant to obtain serum. All serum samples were stored at $-80{ }^{\circ} \mathrm{C}$ until use. After blood withdrawal, mice were intracardially perfused with heparinized phosphatebuffered saline (PBS) to remove remaining blood. After perfusion, the brains and spleens were dissected out and subjected to histopathological examination and/or preparation of single cell population for flow cytometric analysis of immune cells. Five mice for each group at different timepoint post-TBI were used.

\section{Preparation of single-cell population from mouse brain and spleen}

After perfusion with heparinized PBS, mice were sacrificed at various timepoints of post-TBI or post-sham as described above. Excised brains were used to isolate single-cell population as previously described with minor modifications [46]. Briefly, harvested whole brains were cut and minced into small pieces and incubated in $10 \mathrm{~mL}$ Hanks' Balanced Salt Solution (HBSS) with calcium and magnesium supplemented with $1 \mathrm{mg} / \mathrm{mL}$ collagenase $B$ (Sigma-Aldrich, St. Louis, MO) at $37^{\circ} \mathrm{C}$ for $30 \mathrm{~min}$ with intermittent shaking. Clumps of the brain tissues were triturated and filtered through $70 \mu \mathrm{m}$ cell strainers with the addition of $5 \mathrm{~mL}$ of RPMI 1640 medium supplemented with $10 \%$ fetal bovine serum (FBS, Atlanta Biologicals, San Francisco, CA). After spinning at $700 \mathrm{~g}$ for $10 \mathrm{~min}$, the cell pellets were resuspended in $10 \mathrm{~mL}$ of $40 \%$ Percoll gradient (GE Healthcare, Chicago, IL) for centrifugation at $700 \mathrm{~g}$ for $20 \mathrm{~min}$ at room temperature without brake. The cell pellets were washed once with $15 \mathrm{~mL}$ PBS and resuspended in $1 \mathrm{~mL}$ of red blood cell (RBC) lysis buffer (BioLegend, San Diego, CA) for $2 \mathrm{~min}$ at room temperature to lyse RBCs. After centrifugation at $250 \mathrm{~g}$ for $10 \mathrm{~min}$ at $4{ }^{\circ} \mathrm{C}$, cells were harvested for flow cytometric analysis of immune cells in the brain.

Mononuclear cells were also prepared from spleens. Briefly, spleens were mechanically disrupted using the frosted ends of glass slides in $10 \mathrm{~mL}$ of PBS and filtered through $70 \mu \mathrm{m}$ cell strainers, followed by centrifugation at $700 \mathrm{~g}$ for $5 \mathrm{~min}$. Cell pellets were resuspended in $5 \mathrm{~mL} \mathrm{RBC} \mathrm{lysis} \mathrm{buffer} \mathrm{and} \mathrm{incubated} \mathrm{on} \mathrm{ice} \mathrm{for} 5 \mathrm{~min}$, followed by centrifugation at $250 \mathrm{~g}$ for $10 \mathrm{~min}$ at $4{ }^{\circ} \mathrm{C}$ to harvest cells for flow cytometric analysis of immune cells in the spleen.

\section{Flow cytometry}

Single-cell suspensions of brain and spleen tissues were subjected to cell surface staining to determine the frequency and phenotype of immune cells in these tissues. Briefly, cells were first stained with the Zombie Violet ${ }^{\mathrm{TM}}$ Fixable Viability dye (FVD, BioLegend, San Diego, CA) to exclude FVD-positive dead cells and FcR blocking CD16/CD32 Ab (eBioscience, San Diego, CA), followed by staining with fluorochrome-conjugated $\mathrm{Abs}$ against mouse CD45, CD11b, Ly-6G, Ly-6C, F4/80, B220, CD3, CD4, CD8, and PD-1. All Abs were purchased from BioLegend (San Diego, CA). Appropriate isotype controls were used at the same protein concentrations as the test Abs for control staining. After washing, cells were fixed in 2\% PFA and subsequently acquired using a BD LSRFortessa flow cytometer (BD Biosciences, San Jose, CA). Flow data were analyzed using FlowJo v10 software (Tree 
Star, San Carlos, CA). Brain-infiltrating leukocytes were defined as $\mathrm{CD} 45^{\mathrm{High}}$ cells that could be distinguished from CD $45^{\text {Low }}$ glia cells. Within the $\mathrm{CD} 45^{\text {High }}$ population, polymorphonuclear neutrophils (PMNs) were identified by Ly-6G expression, $\mathrm{T}$ cells as $\mathrm{CD} 45^{\mathrm{High}} \mathrm{CD}^{+}$ cells, CD4 $\mathrm{T}$ cells as $\mathrm{CD} 45^{+} \mathrm{CD} 3^{+} \mathrm{CD} 4^{+}$, CD8 $\mathrm{T}$ cells as $\mathrm{CD} 45^{+} \mathrm{CD}^{+}{ }^{+} \mathrm{CD} 8^{+}, \mathrm{B}$ cells as $\mathrm{CD} 45^{+} \mathrm{B} 220^{+}$, and $\mathrm{M} / \mathrm{M} \phi$ as $\mathrm{CD} 45^{+} \mathrm{CD} 11 \mathrm{~b}^{+} \mathrm{Ly}-6 \mathrm{G}^{-} . \mathrm{M} / \mathrm{M} \phi$ were further divided into inflammatory Ly- $6 \mathrm{C}^{\mathrm{High}}$ and tissue-repairing Ly$6 \mathrm{C}^{\text {Low }} \mathrm{F} 4 / 80^{\mathrm{High}}$ subsets.

\section{Western blot analysis}

Mice were sacrificed at $24 \mathrm{~h}, 72 \mathrm{~h}, 1$ week and 2-week post-TBI or -sham. Ipsilateral cortex tissues were collected without fixation and homogenized in the T-PER Tissue Protein Extraction Reagent (Thermo Fisher Scientific, Waltham, MA) plus $1 \times$ Protease Inhibitor Cocktail (Sigma-Aldrich, St. Louis, MO). Tissue lysates were pulse-sonicated for $30 \mathrm{~s}$ and followed by centrifugation at $14,000 \times g$ for $15 \mathrm{~min}$ at $4{ }^{\circ} \mathrm{C}$. Supernatant was harvested and subjected to the BCA Protein Assay to determine the protein concentrations. The extracted proteins were subjected to NuPAGE Novex high-performance electrophoresis (Invitrogen, Carlsbad, CA) on an 8-12\% SDS-PAGE gel, and subsequently blotted onto a polyvinylidene difluoride (PVDF) membrane (Millipore, Billerica, MA). The protein bands were probed with PD-L1 Ab (antiPD-L1 at 1:200 dilution, ab233482, Abcam, Cambridge, MA) and detected using LI-COR Odyssey system (LICOR Biosciences, Lincoln, NS). Anti-mouse $\beta$-tubulin Ab (Abcam, Cambridge, MA) was used as an internal control. For protein expression quantification, immunoreactive bands were scanned and quantified using NIH ImageJ software. The intensity of each PD-L1 band was normalized with the intensity of its corresponding loading control ( $\beta$-tubulin) and plotted as arbitrary units. All Western blots were repeated twice and performed using three mouse brain samples per experimental condition.

\section{Enzyme-linked immunosorbent assay (ELISA)}

The levels of PD-L1 and CCL-2 (also known as monocyte chemoattractant protein-1 or MCP-1) in the brain lysates from mice post-TBI or post-sham and MCP-1 levels in CSF and serum from mice post-sham or TBI were quantified using mouse PD-L1 Duoset ELISA Kit and mouse CCL-2/JE/MCP-1 Duoset ELISA Kit, both from R\&D Systems (Minneapolis, MN), respectively.

\section{CRISPR-Cas9-mediated knockout of PD-L1 from human astrocyte cell line}

PD-L1, also known as B7 homolog 1 (B7-H1) or CD274, represents the first functionally characterized ligand of the inhibitory PD-1 [47]. The PD-L1 gene is highly conserved across species, suggesting its functional importance in many species $[47,48]$. The human PD-L1 gene is comprised of 7 exons including a short $5^{\prime}$ untranslated region (exon 1 ), a secretory signal at the amino terminus (exon 2), an IgV domain (exon 3), an IgC domain (exon 4), a transmembrane domain (exon 5), a short cytoplasmic tail (exon 6), and a long $3^{\prime}$ untranslated region (exon 7) [47-49]. To disrupt the PD-L1 gene at the genome level, we used a single plasmid CRISPR system with a 20-bp guide RNA (gRNA) that was aligned to its target site in exon 3 in the PD-L1 gene (5'-AGCATAGTA GCTACAGACAG-3'), designed by the GUIDES program [50]. Forward and complementary reverse oligos containing a 20-base guide RNA (gRNA) were annealed and cloned into pSpCas9(BB)-2A-GFP (PX458) [51], a backbone plasmid co-expressing Cas9 and EGFP (a gift from Feng Zhang; Addgene plasmid\# 48138), to generate a single plasmid construct co-expressing gRNA, Cas9, and EGFP. The recombinant plasmid clones, each with an insertion of a single gRNA under U6 promotor control, were screened by PCR using the universal hU6 forward primer (5'-GAGGGCCTATTTCCCATGATT-3') and the gRNA reverse primer. The Cas9-gRNA recombinant plasmid $(2.5 \mu \mathrm{g})$ was transfected into U87MG cells, a human astrocyte cell line (ATCC, Manassas, VA), using Lipofectamine 3000 reagents as per manufacturer's instructions (Invitrogen, Carlsbad, CA). After 7 days of culture, PD-L1-negative cells were sorted using flow cytometry. The sorted cells were cultured and followed by an additional sorting of cells with PD-L1 knockout (KO) phenotype.

To study the regulation of PD-L1 expression and MCP-1 production, both wild-type (WT) U87MG and PD-L1 exon $3 \mathrm{KO}$ U87MG cells were treated with medium alone or various combinations of 3 proinflammatory cytokines (IFN- $\gamma$, IL- $1 \beta$, and TNF- $\alpha$ at $10 \mathrm{ng} / \mathrm{mL}$ each cytokine) for $24 \mathrm{~h}$. During the last $6 \mathrm{~h}$ of incubation, brefeldin A (BFA, eBioscience, San Diego, CA) was added to a final concentration of $3 \mu \mathrm{M}$ to allow intracellular accumulation of cytokines. Stimulated cells were stained with fluorochrome-conjugated anti-MCP-1 using the Cytofix/Cytoperm kit (BD Biosciences, San Jose, CA) to determine MCP-1 production. All experiments were repeated at least four times.

\section{Statistical analysis}

The two-sample test for two group comparison and ANOVA for multiple group comparison were used. The generalized linear mixture model (GLMM) was used to fit and test the repeatedly measured longitudinal data of Rotarod followed by post hoc Tukey HSD for pairwise comparison. ANOVA test, followed by Dunnett's test, was used to calculate differences in flow data 
between TBI and sham groups at different timepoints. Two-tailed $t$ test was used to calculate differences in flow data between the anti-PD-L1 treated group and the IgG control group and differences between the wild-type and PD-L1 KO U87MG cell lines. $p<0.05$ was considered statistically significant.

\section{Results}

De novo expression of PD-L1 was markedly and transiently induced in the brain of TBI mice

In general, most ICPs consist of paired receptor-ligand molecules that exert inhibitory or stimulatory effects on immune defense, surveillance, regulation, and self-tolerance [17-21]. The origin, regulation, and function of ICPs have been studied most extensively in the peripheral blood and peripheral tissues, but not in the CNS. Given that the neuroimmune interactions in the brain parenchyma are tightly regulated to prevent overwhelming immune activation in this organ that has little recovery capacity, we hypothesized that there were ICP axes in the CNS, which played a critical role in maintaining the neuroimmune homeostasis. To test this hypothesis, we used TBI mice to explore whether ICP expression could be induced or regulated in the brain in vivo in response to neuroimmune activation, as a sterile immune response develops within minutes in TBI. We performed IHC study of PD-L1 in the brain of TBI versus sham mice. As shown in Fig. 1, PD-L1 expression was barely detectable in sham mice. In TBI mice, de novo expression of PD-L1 was markedly and transiently induced in the brain, particularly in the cortex around the lesion area (Fig. 1A). PD-L1 expression reached the highest level on 3-day to 1-week post-TBI and declined to low or undetectable levels by 2-week post-TBI (Fig. 1A, B). PD-L1 signal was solely found in the injured side of the brain, not in the contralateral side (Fig. 1A). These IHC results were also confirmed by western blot analysis. As shown in Fig. 1C, $\mathrm{D}, \mathrm{PD}-\mathrm{L} 1$ expression was detected in brain tissue lysates from mice on $24 \mathrm{~h}$ to 1 -week post-TBI and became low or undetectable by 2 -week post-TBI. PD-L1 signal was barely detectable in brain tissue lysates from sham mice at any timepoint during a 2-week experiment (Fig. 1AD). Thus, de novo expression of PD-L1 is markedly and transiently induced in response to a sterile immune response in the brain of TBI mice.

\section{PD-L1 expression was transiently and specifically induced in astrocytes in the brain of TBI mice}

Next, we evaluated PD-L1 expression in neural cell types in the CNS. We performed IHC analysis of PD-L1 expression in astrocytes, microglia, neurons, and oligodendrocyte progenitor cells (OPCs) in the brain tissues from TBI versus sham mice. As shown in Fig. 2, PD-L1 expression was transiently and exclusively induced in reactive astrocytes in the brain of TBI mice. PD-L1 expression reached the highest level on 3-day to 1-week

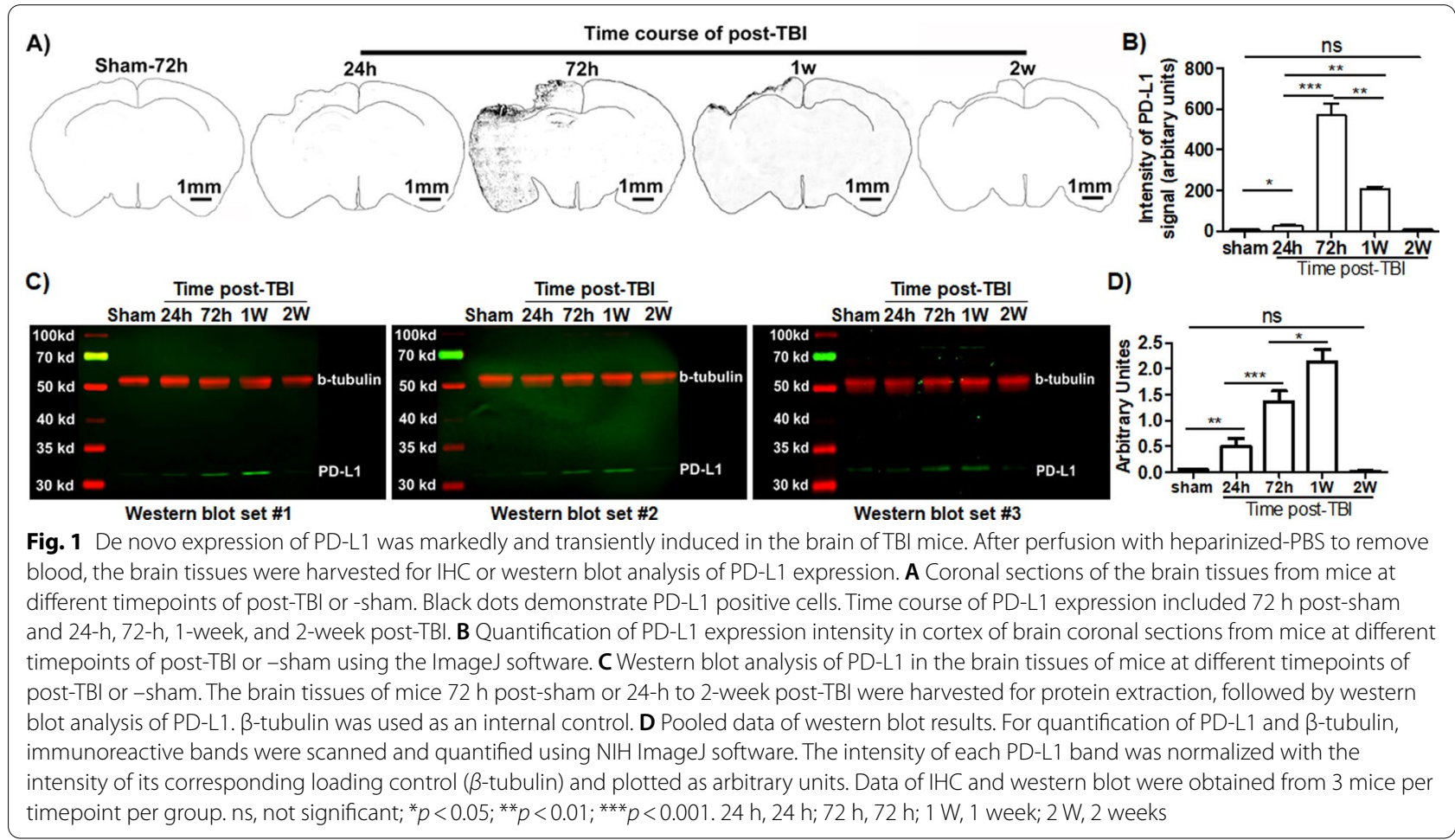



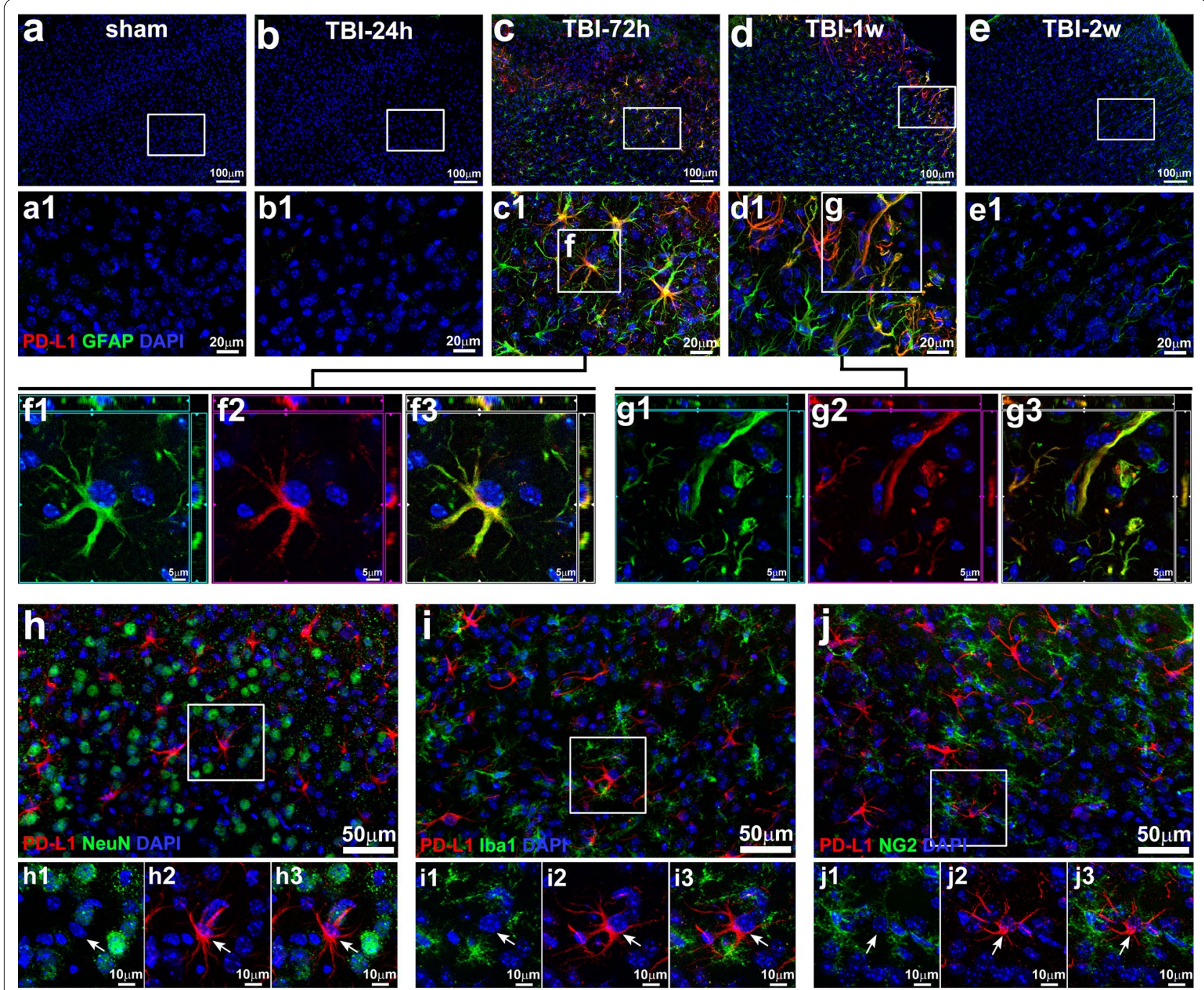

Fig. 2 PD-L1 expression was specifically and transiently induced in astrocytes in TBI mice. Cortex sections of the brains from sham mice (a, a1) and TBI mice at different timepoints of post-injury, $24 \mathrm{~h}(\mathbf{b}, \mathbf{b} \mathbf{1}), 72 \mathrm{~h}(\mathbf{c}, \mathbf{c} \mathbf{1}), 1 \mathrm{w}(\mathbf{d}, \mathbf{d} \mathbf{1})$ and $2 \mathrm{~W}(\mathbf{e}, \mathbf{e} \mathbf{1})$ were stained with antibodies against PD-L1 (red) and GFAP (green for astrocytes). Enlarged single cell images of the $\mathbf{c} \mathbf{1 - f}(\mathbf{f} \mathbf{1}-\mathbf{3})$ and $\mathbf{d} \mathbf{1}-\mathbf{g}(\mathbf{g} \mathbf{1}-\mathbf{3})$ as highlighted by the white box showed the Co-localization of PD-L1 with GFAP in reactive astrocytes. $\mathbf{h}-\mathbf{j}$, low power image of IHC staining of the brain tissue sections with antibodies against PD-L1 (red) and different neural cell type specific markers (green) including NeuN (neurons), Iba1 (microglia), and NG2 (oligodendrocyte progenitor cells or OPCs). DAPI (blue) was used for counter staining nucleus in these IHC analyses. High power images showed no PD-L1 expression was detected in either Neuron (h1-3), reactive microglia (i1-3), or OPCs (j1-3)

post-TBI in reactive astrocytes, where PD-L1 and GFAP were co-localized (Fig. 2c, c1, d, and d1). The co-expression of PD-L1 and GFAP was clearly visualized in high power images (Fig. 2f1-3 and g1-3). Thereafter, PD-L1 expression declined and became low or undetectable by 2-week post-TBI (Fig. 2e and e1). PD-L1 expression was undetectable in NeuN-positive neurons (Fig. 2h, h1-3), Iba1-positve microglia (Fig. 2i, i1-3), and NG2-positive OPCs (Fig. 2j, j1-3). Thus, de novo expression of PD-L1 was markedly and selectively induced in astrocytes, not in other types of neural cells in the brain of TBI mice.
Under normal physiological circumstances, astrocytes in the adult brain are quiescent and non-migratory cells [52]. However, astrocytes can be activated to become migratory under pathological conditions, such as TBI and neuroinflammation [52]. We found that the distribution of $\mathrm{PD}-\mathrm{L1}^{+}$reactive astrocytes was changing during the course of TBI. As shown in Figs. 1A and 3, widespread $\mathrm{PD}-\mathrm{L}^{+}{ }^{+}$reactive astrocytes were observed in the injured cortex with highest density close to the edge of the cavity on day 3 post-TBI. Quantification of the percentage of $\mathrm{PD}-\mathrm{L}^{+}{ }^{+}$reactive astrocytes $\left(\mathrm{PD}-\mathrm{L}^{+}{ }^{+} \mathrm{GFAP}^{+}\right)$ 


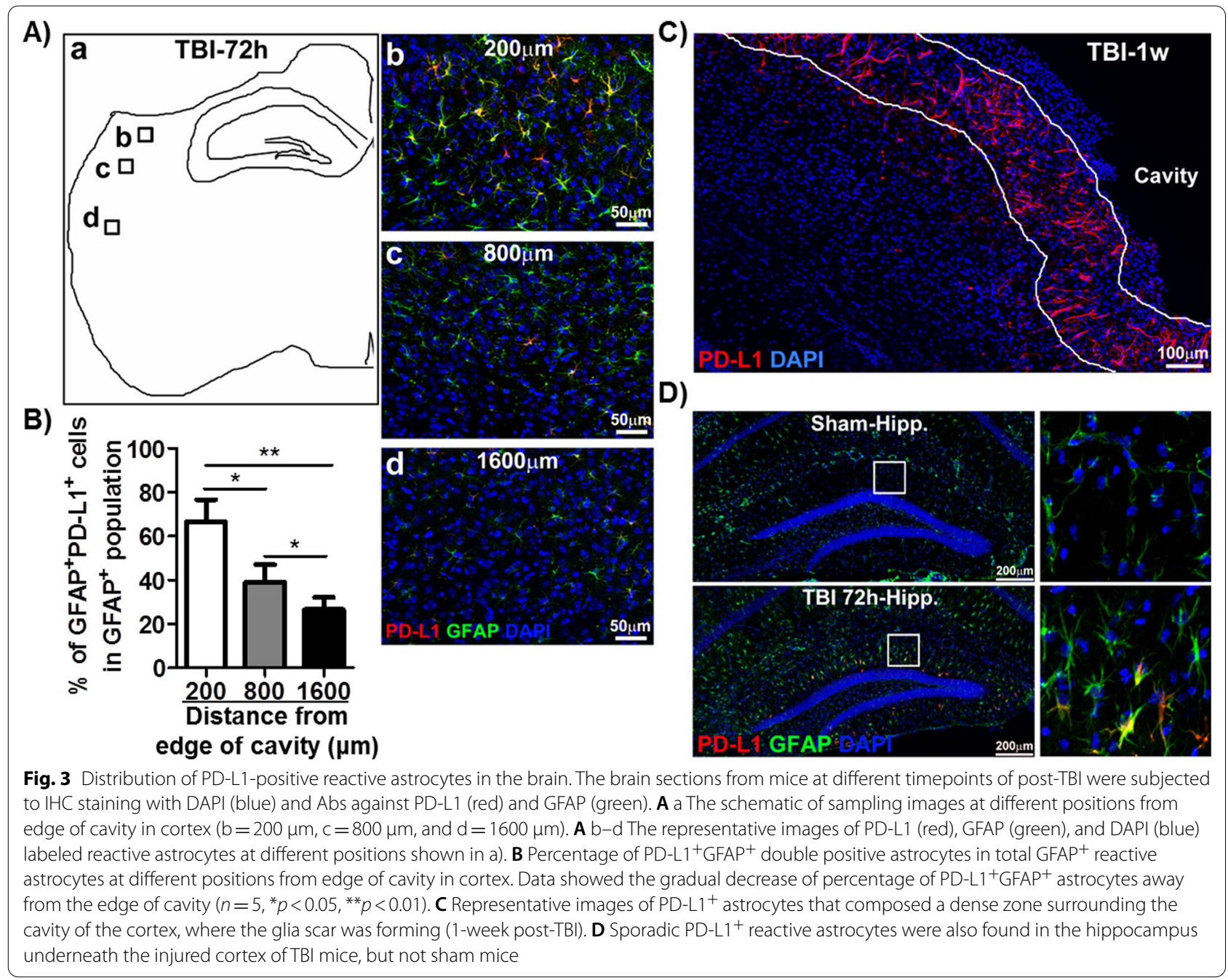

in total GFAP-positive cells at different distances from the injury site showed that approximately $80 \%$ of the reactive astrocytes expressed PD-L1 within $200 \mu \mathrm{m}$ to the injured site (Fig. 3B). The percentage of $\mathrm{PD}-\mathrm{L1}^{+}$reactive astrocytes gradually decreased with an increase in the distance away from the injured site (from $\sim 80 \% / 200 \mu \mathrm{m}$ to $\sim 30 \% / 1600 \mu \mathrm{m}$, Fig. 3B). Strikingly, by 1 -week post$\mathrm{TBI}, \mathrm{PD}-\mathrm{L}^{+}$reactive astrocytes were highly enriched to form a dense zone around the TBI lesion (Fig. 3C), where the glial scar was forming. In addition, most PD-L1 ${ }^{+}$cells at this time exhibited scar forming astrocyte-like morphology with long and thin extensions toward the injured site (Fig. 2, d1). Notably, sporadic PD- $1^{+}$astrocytes were also observed in the hippocampus of TBI mice at day 3, although the frequency was low (Fig. 3D). These results suggest that $\mathrm{PD}-\mathrm{L}^{+}$reactive astrocytes may be involved in glial scar formation to confine TBI-triggered brain damage.
Taken together, de novo expression of PD-L1 was markedly and selectively induced in astrocytes, but not in neurons, microglia, or OPCs, and PD-L1 ${ }^{+}$astrocytes were highly enriched to form a dense zone around the lesion site in TBI mice.

\section{Blockade of PD-L1 increased brain tissue cavity and worsened functional outcomes of TBI}

Reactive astrocytes can exert biphasic functions, that is, beneficial and detrimental effects in the CNS, yet the mechanisms remain largely unclear. To clarify whether $\mathrm{PD}-\mathrm{L}^{+}$reactive astrocytes played a beneficial or detrimental role, we applied a single dose of PD-L1 blocking $\mathrm{Ab}$ via $S C$ injection to mice $24 \mathrm{~h}$ post-TBI. To verify whether PD-L1 Ab arrived at the injured site of TBI brain and was bound to reactive astrocytes, PD-L1-Alex 647-conjugated $\mathrm{Ab}$ was separately given via $S C$ injection. After $48 \mathrm{~h}$ of $S C$ injection, the brain tissue was collected 
(72 $\mathrm{h}$ post-TBI) and directly examined via microscopy without additional immunostaining. The result confirmed that PD-L1-Alex 647-conjugated Ab was bound to GFAP-positive reactive astrocytes in the injured cortex (Additional file 1: Fig. S1). We also quantified the GFAP reactivation after $\mathrm{PD}-\mathrm{L} 1 \mathrm{Ab}$ treatment and compared to that in IgG control group. There was no significant difference between these two groups, indicating treatment of anti-PD-L1 Ab does not affect astrocyte reaction (Additional file 1: Fig. S2).

To explore whether PD-L1 blockade affected the outcomes of TBI, we performed IHC to analyze brain tissue damage and used the rotarod and EPM tests to evaluate motor function and anxiety-related behavior, respectively, as described in our previous reports [53]. As shown in Fig. 4A, B, there was no obvious cavity in ipsilateral cortex of sham mice with or without PD-L1 $\mathrm{Ab}$ treatment. In contrast, a larger cavity size was present in the cortex of TBI mice with PD-L1 Ab treatment when compared with IgG treatment (Fig. 4A, B). For the rotarod test, mice were randomly divided into 4 groups 1 day prior to TBI or sham. These mice were given a rotarod test to collect baseline data of mouse motor function. As shown in Fig. 4C (day 0), there was no significant

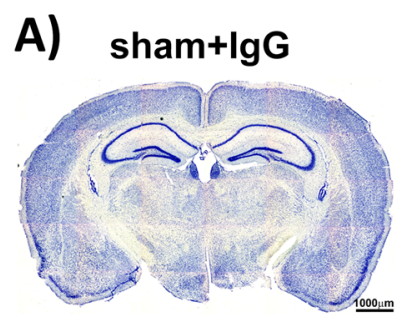

sham+PD-L1 Ab
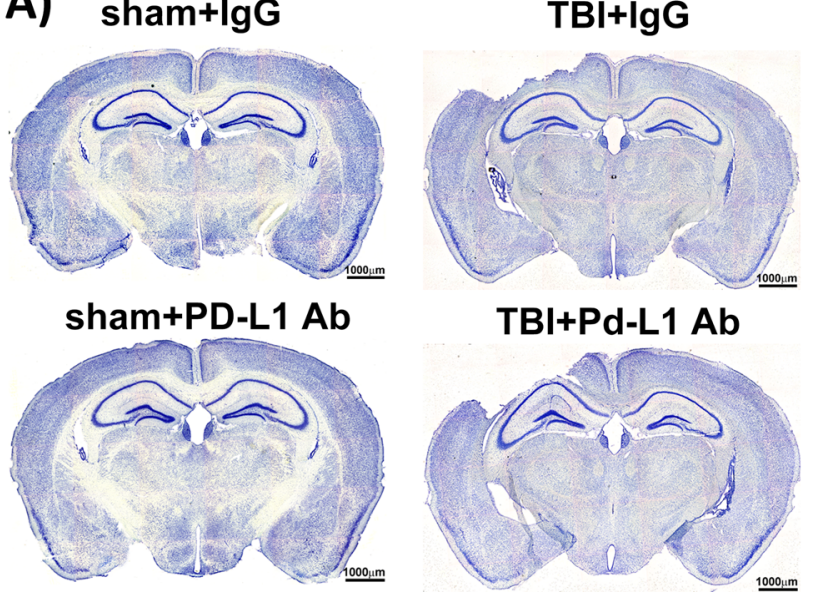

$\mathrm{TBI}+\mathrm{Pd}-\mathrm{L} 1 \mathrm{Ab}$

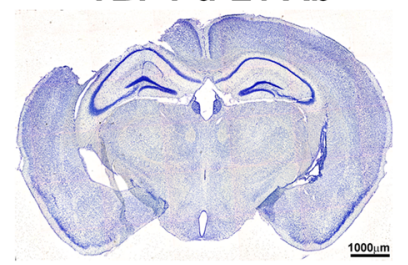

B)

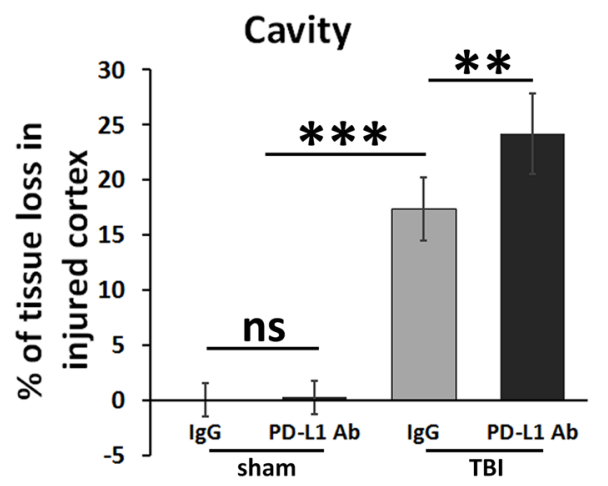

C) Motor function

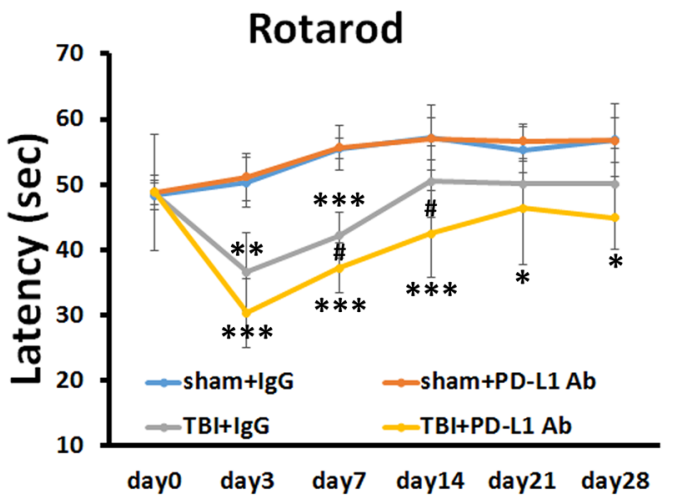

D) Emotion: impulsivity EPM
Open arm : time

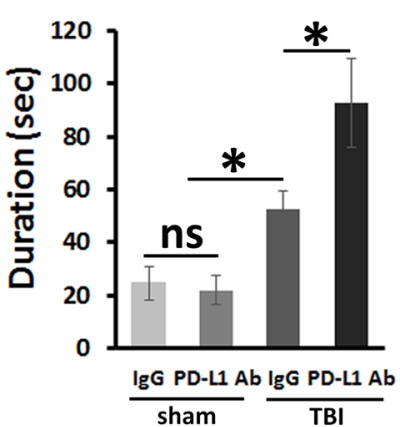

Open arm : distance

Fig. 4 Blockade of PD-L1 increased the cavity size of injured cortex and motor and emotion dysfunction of TBI mice. After $24 \mathrm{~h}$ of surgery, each mouse was given a single dose $(200 \mathrm{\mu g} / \mathrm{kg})$ of anti-PD-L1 Ab or lgG via subcutaneous injection. A Representative images of the brain of sham mice and tissue cavity in injured cortex of TBI mice treated with PD-L1 Ab or lgG using the Nissl staining. B Quantitative data of tissue loss in ipsilateral cortex of sham and TBI mice with PD-L1 Ab or lgG treatment ( $n=5 /$ group). $C$ Rotarod test was conducted to determine the motor function. Mice received rotarod test for baseline 1 day before sugery and at days $3,7,14,21$, and 28 post-TBI or post-sham. Data were presented as average \pm SD and analyzed using the repeated measures ANOVA followed by post hoc Tukey HSD ( $n=10 /$ group). D The elevated plus maze (EPM) consisting of open and enclosed arms was used to determine mouse anxious and impulsive behavior. The time (Left) and distance (Right) of each mouse spent on the open arms were recorded and calculated. Data were presented as average \pm SD and analyzed using the two way ANOVA ( $n=10 /$ group). ${ }^{*}$ comparison of TBI versus sham, \#comparison of PD-L1 Ab versus IgG in TBI. ns, not significant; ${ }^{*} p<0.05,{ }^{*} p<0.05 ;{ }^{* *} p<0.01$; ${ }^{* * *} p<0.001$ 
difference of motor function at baseline prior to craniotomy between the groups of mice. Following treatment with IgG or PD-L1 blocking Ab, mice underwent the rotarod test 4 times per timepoint at days 3, 7, 14, 21, and 28 post-TBI or post-sham. The amount of time (latency to fall) that the mice stayed on the rod was recorded and anaylzed. PD-L1 Ab treatment did not affect the motor function in sham mice. While, TBI rapidly and significantly impaired the motor function of injured mice compared to sham control, as the latency on rod at day 3 post-TBI was dramatically decreased $(\mathrm{TBI}+\mathrm{IgG}$ at $36.7 \pm 5.9 \mathrm{~s}$ versus sham + IgG at $50.4 \pm 3.8 \mathrm{~s}, p<0.001$, $n=10$ /group). The motor function of TBI mice gradually improved until there was not significant difference when compared to sham controls at day 14 post-injury (TBI at $50.5 \pm 5.5 \mathrm{~s}$ versus sham at $56.5 \pm 4.1 \mathrm{~s}, p>0.05$ ). PD-L1 blocking Ab administration not only aggravated the motor function deficit in TBI mice but also prolonged the motor function recovery (Fig. 4C). PD-L1 blockade further decreased the latency on rod at day 3 postTBI (TBI+PD-L1 Ab at $30.3 \pm 5.3 \mathrm{~s}$ versus $\mathrm{TBI}+\mathrm{IgG}$ at $36.7 \pm 5.9 \mathrm{~s}, p<0.05, n=10 /$ group) (Fig. $4 \mathrm{C}$ ). The heightened motor function impairment in TBI mice with PD-L1 blockade persisted through the entire experimental course of 28 days, remaining impaired compared to sham mice even at day 28 post-TBI (TBI + PD-L1 Ab at $44.9 \pm 4.9 \mathrm{~s}$ versus sham $+\mathrm{PD}-\mathrm{L} 1 \mathrm{Ab}$ at $56.8 \pm 3.4 \mathrm{~s}$, $p<0.05, n=10 /$ group) (Fig. 4C).

Since PD-L1 blockade induced prolonged impairment of motor functon, we further performed EPM tests to assess the effects of PD-L1 blockade on anxietyrelated behavior, one of hippocamus-related emotional deficit, on day 29 post-TBI (28 days after PD-L1 Ab treatment). Same as rotarod test, PD-L1 Ab treatment did not change the behavior of sham mice comapring to IgG treatment (Fig. 4D). In agreement with previous findings [44], TBI mice exhibited reduced anxiety, spending more time (TBI+IgG at $47.5 \pm 11.2 \mathrm{~s}$ versus sham $+\operatorname{IgG}$ at $24.2 \pm 6.4, p<0.05, n=10$ /group) and traveling further (TBI+ IgG at $1.1 \pm 0.36 \mathrm{~m}$ versus sham + IgG at $0.35 \pm 0.09 \mathrm{~m}, p<0.05, n=10$ /group) in the open arms of the EPM when compared to sham mice (Fig. 4D). TBI mice with PD-L1 blockade spent more time (TBI+PD-L1 Ab: $90.4 \pm 17.8 \mathrm{~s}$ versus $\mathrm{TBI}+\mathrm{IgG}$ : $47.5 \pm 11.2 \mathrm{~s}, p<0.05, n=10$ /group) and traveled further (TBI+PD-L1 Ab: $1.80 \pm 0.32 \mathrm{~m}$ versus TBI+IgG: $1.1 \pm 0.36 \mathrm{~m}, p<0.05, n=10$ /group) in open arms when compared to IgG-treated mice (Fig. 4D). Of note, the movement speed of animals was not significantly different between the groups (data not shown).

Taken together, PD-L1 blockade increased the size of the brain tissue cavity, exacerbated the motor function impairment, and changed anxiety-related behavior, suggesting that PD-L1 signaling in astrocytes plays a critical role in maintaining CNS tissue integrity and functional outcomes after TBI.

\section{Blockade of PD-L1 prolonged the accumulation of inflammatory Ly- $6 \mathrm{C}^{\mathrm{High}}$, but not tissue-repairing Ly- $6 C^{\text {Low }} \mathrm{F} 4 / 80^{+} \mathrm{M} / \mathrm{M} \varphi$ in the brain of TBI mice}

To study the mechanisms underlying worse outcomes of TBI due to PD-L1 blockade, we analyzed and compared immune cell infiltration to the brain versus spleen. First, we analyzed the frequency and phenotype of immune cells in the brain and spleen from heparinized PBS-perfused mice post-TBI versus postsham at various timepoints. Figure $5 \mathrm{~A}$ shows the flow cytometry gating strategies for analyzing the immune cells in the brain. We found that the profile and frequency of immune cells were markedly altered in the brain, but less in the spleen. The percentages of neutrophils $\left(\mathrm{CD} 45^{+} \mathrm{Ly}^{-6 \mathrm{G}^{+}}\right)$, B cells $\left(\mathrm{CD} 45^{+} \mathrm{CD} 3^{-} \mathrm{B} 220^{+}\right)$, and $\mathrm{M} / \mathrm{M} \phi\left(\mathrm{CD} 45^{\mathrm{High}} \mathrm{Ly}-6 \mathrm{G}^{-} \mathrm{CD} 11 \mathrm{~b}^{+}\right)$were increased in the brain, while the percentages of $\mathrm{T}$ cells including total $\mathrm{T}$ cells $\left(\mathrm{CD}^{+}\right), \mathrm{CD} 4 \mathrm{~T}$ cells $\left(\mathrm{CD}^{+}{ }^{+} \mathrm{CD} 4^{+}\right)$, and $\mathrm{CD} 8 \mathrm{~T}$ cells $\left(\mathrm{CD}^{+} \mathrm{CD}^{+}\right)$remained unchanged (data

\footnotetext{
(See figure on next page.)

Fig. 5 Effects of PD-L1 blockade on migration of immune cells to the brain of TBI mice. After 7 days of TBI (6 days of PD-L1 blockade), whole brain was harvested from each mouse after removal of blood cells by perfusion with heparinized-PBS, followed by preparation of brain single-cell suspension. Cells were stained with the Zombie Violet ${ }^{\mathrm{TM}}$ FVD to exclude FVD-positive dead cells, followed by incubation with CD16/ CD32 Abs for blocking FcR binding, and fluorochrome-conjugated Abs against mouse CD45, CD3, CD4, CD8, CD11b, Ly-6G, Ly-6C, F4/80, B220, and PD-1. Brain-infiltrating leukocytes (LYM) were defined as CD45 ${ }^{\text {High }}$ cells that could be distinguished from CD45 ${ }^{\text {Low }}$ glia cells. Within the CD45 ${ }^{\text {High }}$ population, polymorphonuclear neutrophils (PMNs) were identified by Ly-6G expression, T cells as CD45 ${ }^{\text {High }}$ CD3 ${ }^{+}$cells, B cells as $\mathrm{CD}_{4}{ }^{+} \mathrm{B} 220^{+}$, and M/M $\phi$ as CD45 ${ }^{+} \mathrm{CD} 11 \mathrm{~b}^{+} \mathrm{Ly}^{-6 G^{-}}$. M/M $\phi$ were further divided into Ly- $6 \mathrm{C}^{\text {High }}$ and Ly- $6 \mathrm{C}^{\text {Low }} \mathrm{F} 4 / 80^{\text {High }}$ subsets. A Gating strategy for flow cytometric analysis of immune cells in the brain of TBI mice. B Representative flow plots of brain cells from the gated $\mathrm{M} / \mathrm{M} \varphi$ (M) showing $\%$ of Ly- $6 C^{\text {High }}$ versus Ly- $6 C^{\text {Low }}$ $4 / 80^{\text {High }} \mathrm{M} / \mathrm{M} \varphi$ in the brain of TBI mice treated with PD-L1 Ab versus IgG. C Pooled data of Ly- $6 C^{\text {High }}$ versus Ly-6C ${ }^{\text {Low }} \mathrm{F} 4 / 80^{\text {High }} \mathrm{M} / \mathrm{M} \varphi$ in the brain of TBI mice treated with PD-L1 Ab versus lgG $(n=5) .{ }^{*} p<0.05$. D Representative flow plots showing PD-1 expressing T cells. E Representative IHC of CD45+ leukocytes (red) in injured cortex from mice treated with PD-L1 Ab versus IgG ( $n=5$ ). F ELISA results of MCP-1 in the brain lysates $(n=3)$, MCP-1 in CSF and serum samples $(n=5)$. G The levels of PD-L1 and MCP-1 in the brain of TBI mice were present at opposite patterns of regulation. ANOVA test with Dunn's corrections was used for comparing sham versus TBI. ns, not significant; ${ }^{*} p<0.05 ;{ }^{* * *} p<0.001$
} 


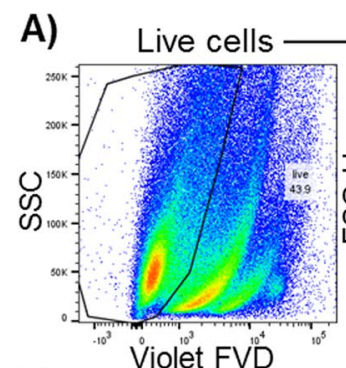

B) $\mathrm{TBI}+\mathrm{PD}-\mathrm{L} 1 \mathrm{Ab}$
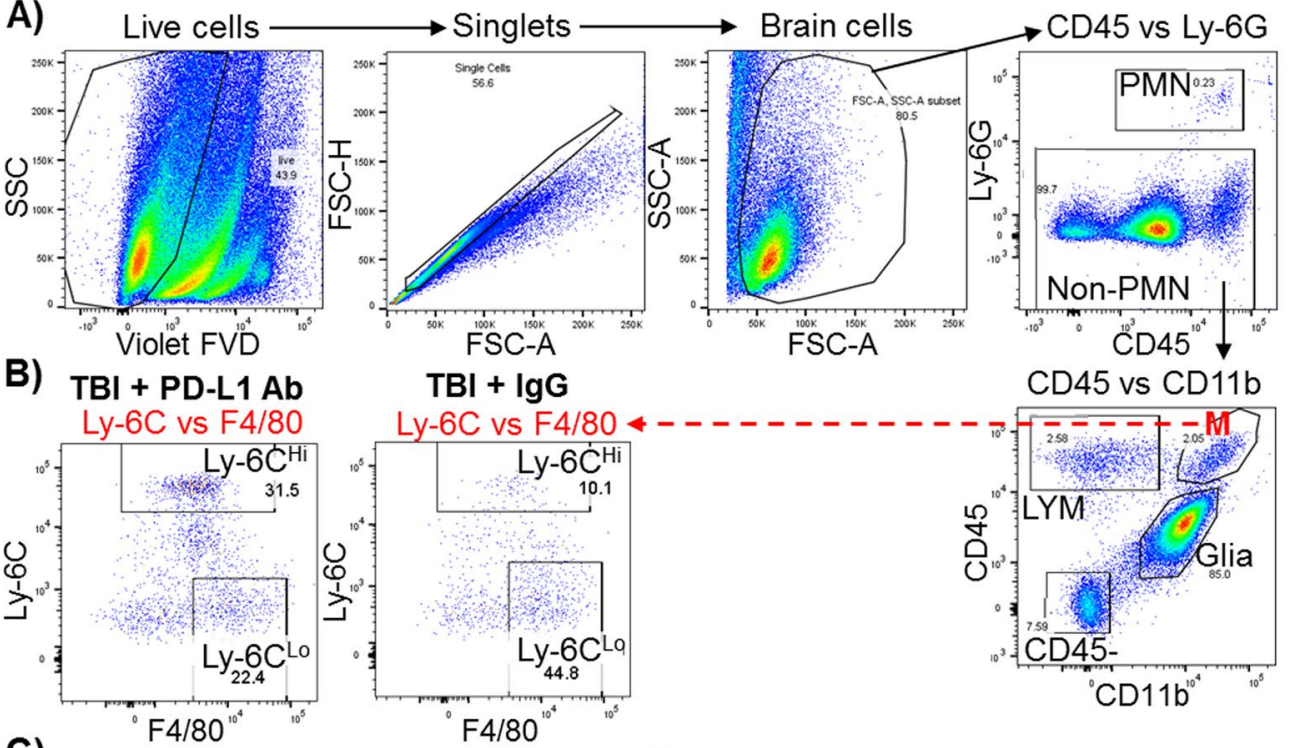

C)

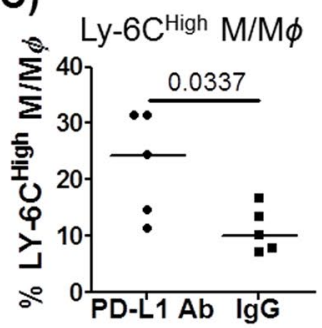

E)

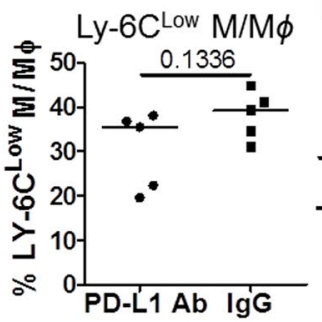

D
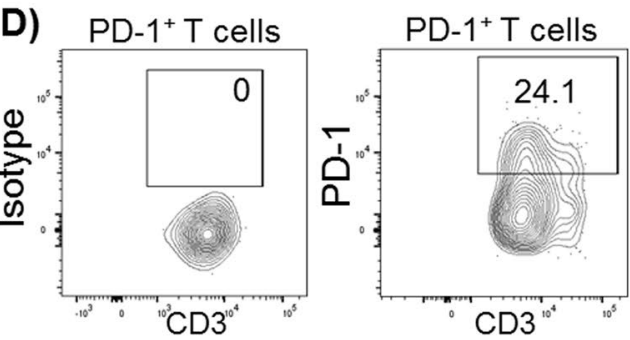

TBI + IgG

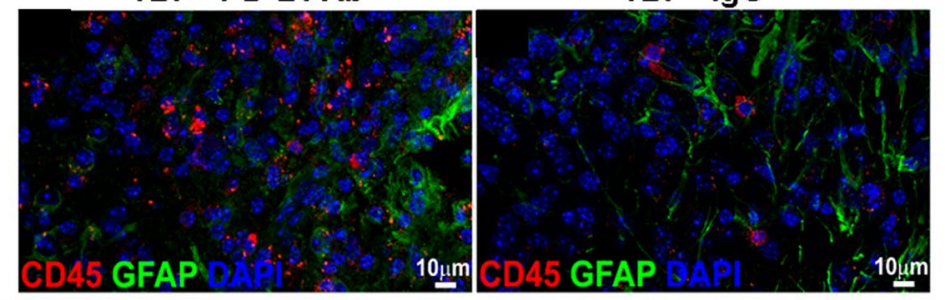

F)

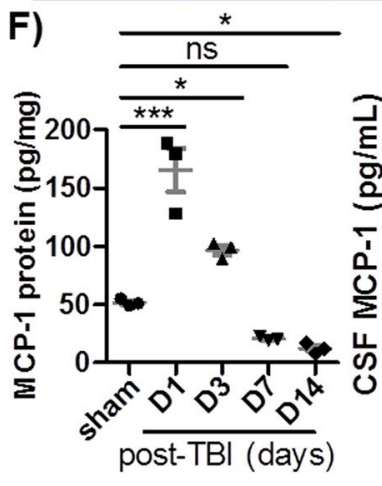

Fig. 5 (See legend on previous page.)
G)

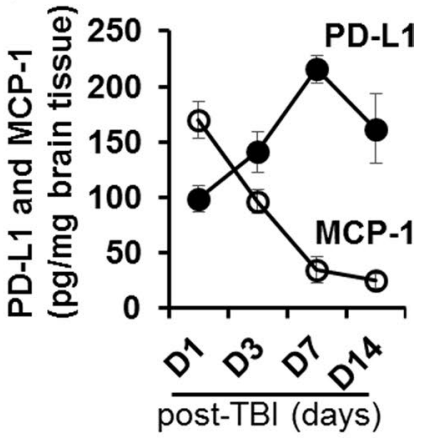

not shown). The neutrophils and B cells in the brain peaked at $24 \mathrm{~h}$ post-TBI, while total $\mathrm{M} / \mathrm{M} \phi$ reached their peak at $72 \mathrm{~h}$ post-TBI (data not shown). The accumulated $\mathrm{M} / \mathrm{M} \phi$ in the brain were dominated by Ly-6 $C^{\text {High }} \mathrm{M} / \mathrm{M} \phi\left(\mathrm{CD} 45^{\text {High }} \mathrm{Ly} 6-\mathrm{G}^{-} \mathrm{CD} 11 \mathrm{~b}^{+} \mathrm{Ly}-6 \mathrm{C}^{\text {High }}\right)$ that were increased in a pattern similar to the 
total $\mathrm{M} / \mathrm{M} \phi$. In contrast, Ly- $6 \mathrm{C}^{\mathrm{Low}} \mathrm{F} 40 / 80^{+} \mathrm{M} / \mathrm{M} \phi$ $\left(\mathrm{CD} 45^{\text {High }}\right.$ Ly6-G ${ }^{-} \mathrm{CD} 11 \mathrm{~b}^{+}$Ly-6C ${ }^{\text {Low }} \mathrm{F} 4 / 80^{+}$) decreased and reached their lowest percentages at $72 \mathrm{~h}$ post-TBI (data not shown). By day 7 post-TBI, all alterations described above returned to the levels found in sham mice (data not shown). These types of immune cells in the spleen were not dramatically altered except for the Ly- $6 C^{\text {High }} \mathrm{M} / \mathrm{M} \phi$ that were decreased at 24 h post-TBI (data not shown). Therefore, immune cells in the CNS and peripheral lymphoid tissues exhibit different patterns of immune responses to TBI. Particularly, TBI triggers an increase of inflammatory Ly- $6 C^{\text {High }} M / M \phi$, but a decrease of tissue-repairing Ly- $6 C^{\text {Low }} \mathrm{M} / \mathrm{M} \phi$ in the brain. The increased Ly- $6 C^{\text {High }} M / M \phi$ are likely derived from the migration of compartments from peripheral lymphoid tissues, such as spleen, because the splenic Ly- $6 C^{\text {High }} M / M \phi$ population seems to be inversely related to the brain population.

Next, we studied whether PD-L1 signaling affected the infiltration of immune cells to the brain of TBI versus sham mice. We found that PD-L1 blockade prolonged the accumulation of inflammatory Ly- $6 \mathrm{C}^{\text {High }}$ $\mathrm{M} / \mathrm{M} \phi$ in the brain of TBI mice, while the percentage of tissue-repairing Ly-6 $\mathrm{C}^{\mathrm{Low}} \mathrm{M} / \mathrm{M} \phi$ was decreased, albeit not to a statistical significance, in the brain of TBI mice (Fig. 5B, C). Other types of immune cells in the brain and spleen were not significantly affected by PD-L1 blockade. Notably, $25 \%$ of total T cells in the brain of TBI mice were PD-1 ${ }^{+}$(Fig. 5D), which likely represent a major trigger of PD-1/PD-L1 signals. IHC assays also revealed that PD-L1 blockade increased $\mathrm{CD} 45^{+}$leukocytes in the injured cortex (Fig. 5E).

Since MCP-1 (also known as CCL2) is the principal chemokine for recruiting Ly- $6 \mathrm{C}^{\mathrm{High}} \mathrm{M} / \mathrm{M} \phi$ into inflammatory sites through binding its cognate CCR2 receptor on the cell surface $[54,55]$, we studied the association between levels of PD-L1 and MCP-1 in the brain of TBI mice. We used ELISA assays to quantitatively measure PD-L1 in the lysates of brain tissues from post-TBI mice, and MCP-1 in the lysates of brain tissues, CSF, and serum from mice post-sham or TBI. We found that basal levels of MCP-1 were detected in the lysates of brain tissues, CSF, and serum from mice post-sham or TBI (Fig. 5F). MCP-1 levels in the lysates of brain tissues and CSF were rapidly increased in TBI mice and reached the highest levels on day 1 post-TBI, while MCP-1 levels in the serum samples did not show any significant changes (Fig. 5F). Interestingly, we found that up-regulation of PD-L1 was associated with decline of MCP-1 in the brain of TBI mice (Fig. 5G). Thus, levels of PD-L1 and MCP-1 in the brain of TBI mice exhibit an opposite pattern of regulation, suggesting that PD-L1 may down-regulate MCP-1 production.
Taken together, PD-L1 blockade increases migration of inflammatory Ly- $6 \mathrm{C}^{\text {High }} \mathrm{M} / \mathrm{M} \phi$ to the brain of TBI mice, which is likely linked to abrogation of PDL1-mediated suppression of MCP-1 production. Thus, $\mathrm{PD}-\mathrm{L} 1^{+}$reactive astrocytes likely act as a brake on neuroinflammation in TBI.

\section{The effects of PD-L1 intrinsic signaling in astrocytes on the production of MCP-1 (CCL2), a chemokine that recruits inflammatory Ly- $6 C^{\text {High }} M / M \varphi$}

PD-L1, a key inhibitory ICP, exerts dual inhibitory signals to suppress the activity of PD-1-expressing $\mathrm{T}$ cells via the PD-1/PD-L1 axis and the function of PD-L1 ${ }^{+}$cells via a novel intrinsic signaling mechanism [21, 30, 56-72]. To test the regulatory role of PD-L1 intrinsic signaling in neuroinflammation, we used CRISPR-Cas9 technology to generate PD-L1 exon 3 knockout (KO) U87MG cells, a human astrocyte cell line (Fig. 6A). As shown in Fig. 6B, wild-type (WT) U87MG cells constitutively expressed PD-L1 on the cell surface, which was not observed in primary astrocytes in the brain of mice. WT U87MG cells increased PD-L1 expression in response to stimulation with IFN- $\gamma$, which was further enhanced by TNF- $\alpha$ and IL-1 $\beta$ (Fig. 6B). After knockout of the PD-L1 gene at the genome level, U87MG cells completely lost PD-L1 expression in the presence or absence of cytokine stimulators (Fig. 6C).

Next, we used PD-L1 KO versus WT U87MG cells to study the effects of PD-L1 intrinsic signaling in astrocytes on the production of MCP-1, a principal chemokine for recruiting Ly- $6 \mathrm{C}^{\mathrm{High}} \mathrm{M} / \mathrm{M} \phi$ through binding to the CCR2 receptor on the cell surface $[54,55]$. At baseline without stimulation, both PD-L1 KO and WT U87MG cells produced little to no MCP-1 (Fig. 6D, upper panel). Stimulation with a mixture of cytokines (IFN- $\gamma$, TNF- $\alpha$, and IL-1 $\beta$ ) markedly increased MCP-1 production (Fig. 6D, lower panel). Notably, the percentage of MCP-1-positive cells was significantly higher in PD-L1 KO than in WT U87MG cells upon stimulation with the mixture of cytokines (Fig. 6D, E). Thus, abrogation of PD-L1 intrinsic signaling in reactive astrocytes enhances MCP-1 production, which is likely linked to increased Ly-6C $\mathrm{C}^{\text {High }}$ $\mathrm{M} / \mathrm{M} \phi$ recruitment into the brain of TBI after PD-L1 blockade.

\section{Discussion}

This report provides evidence showing that ICPs are important and essential components of the neuroimmune system in the brain and play a critical role in the regulation of neuroimmune and neuroinflammatory responses in the brain of TBI mice. We found that de novo expression of PD-L1, a key inhibitory ICP that interacts with its 


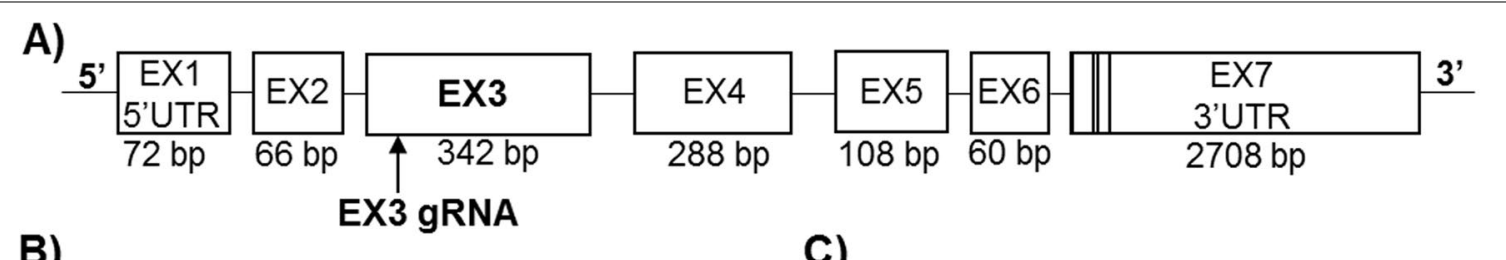

B)

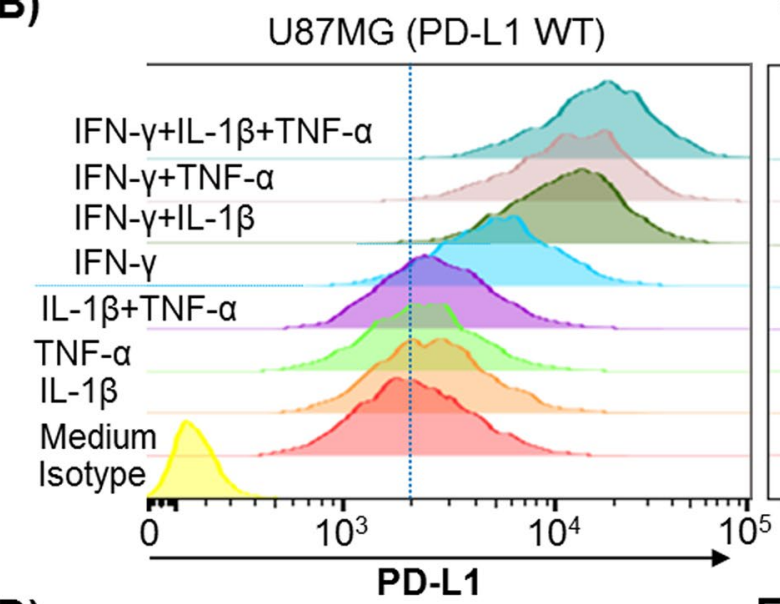

C) U87MG (PD-L1 KO)

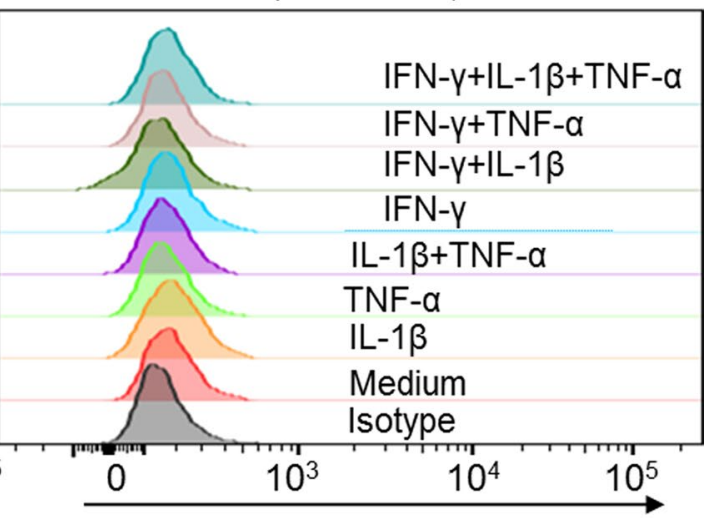

D)

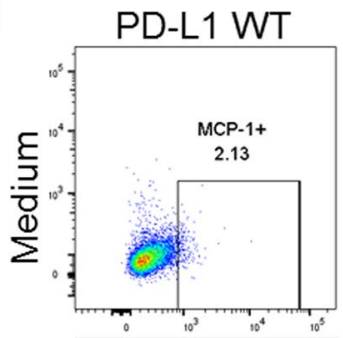

PD-L1 KO

E)
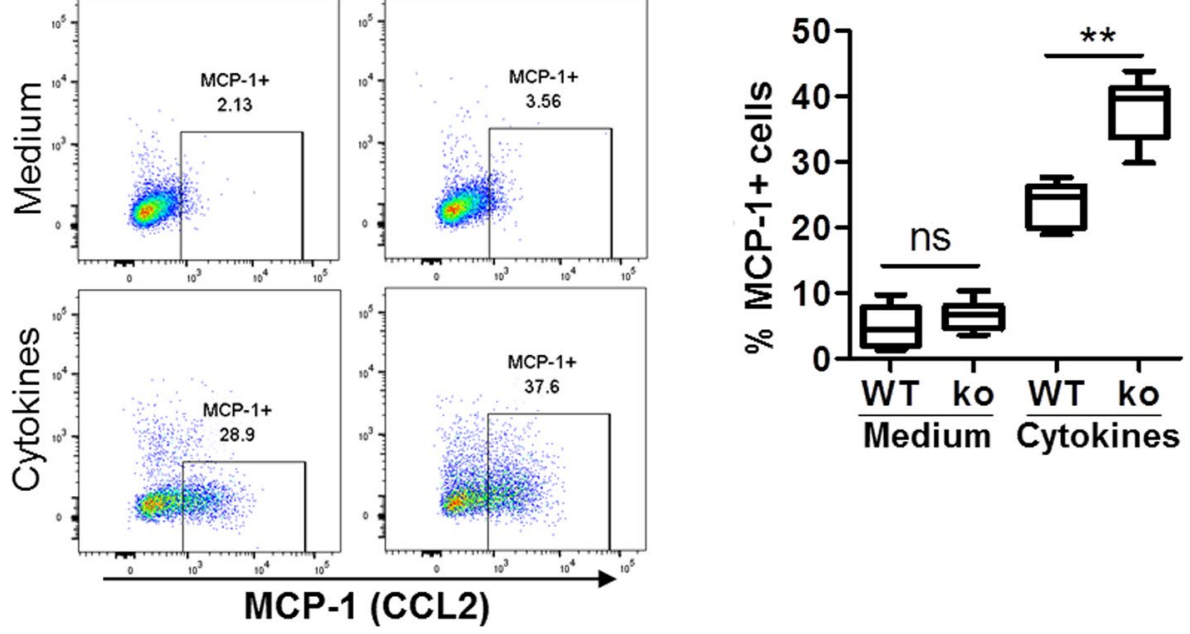

Fig. 6 Effects of PD-L1 knockout from human astrocyte cells (U87MG) on MCP-1 (CCL2) production. A Schematic representation of human PD-L1 gene structure consisting of 7 exons (EX1-EX7, NM_014143.3) and the 20 bp guide RNA (gRNA) that was aligned to its target site in exon 3. B and C Representative histograms of PD-L1 expression from WT U87MG cells versus PD-L1 exon-3 KO U87MG cells. WT U87MG and PD-L1 exon-3 KO U87MG cells were treated with medium, IL-1 $\beta$, TNF-a, IFN- $\gamma$, or their combinations for $24 \mathrm{~h}$ as indicated. Each of these cytokines was used at $10 \mathrm{ng} /$ $\mathrm{mL}$. Cells were subjected to surface staining of PD-L1. D Representative plots of flow cytometry showing the effects of PD-L1 KO on the production of MCP-1. WT U87MG and PD-L1 exon-3 KO U87MG cells were treated with medium or a mixture of cytokines (IFN- $\gamma$, IL-1 3 , and TNF-a at $10 \mathrm{ng} / \mathrm{mL}$ each) for $24 \mathrm{~h}$. Cells were subjected to intracellular staining of MCP-1 to determine MCP-1 production. E Pooled data showing the effects of the effects of PD-L1 KO on the production of MCP-1 in response to stimulation with the mixture of cytokines. All experiments were repeated at least four times. ns, not significant; ${ }^{* *} p<0.01$

cognate receptor PD-1 on the surface of T cells to impede $\mathrm{T}$ cell immunity against tumors and infectious pathogens [21, 30, 56-67], was robustly induced in reactive astrocytes, but not in $\mathrm{Iba}^{+}$microglia, $\mathrm{NeuN}^{+}$neurons, or $\mathrm{NG}^{+}$OPCs (Figs. 1, 2). These PD-L1 ${ }^{+}$astrocytes were highly enriched to form a dense zone around the TBI lesion (Fig. 3). Blockade of PD-L1 signaling enlarged brain tissue cavity size, increased infiltration of inflammatory Ly- $6 \mathrm{C}^{\text {High }} \mathrm{M} / \mathrm{M} \phi$ but not tissue-repairing Ly$6 \mathrm{C}^{\mathrm{Low}} \mathrm{F} 4 / 80^{+} \mathrm{M} / \mathrm{M} \phi$, and worsened TBI outcomes in TBI mice (Figs. 4, 5). Mechanistically, PD-L1 signaling in astrocytes likely exhibited dual inhibitory activities in 
prevention of excessive neuroimmune and neuroinflammatory responses to TBI through (1) the PD-1/PD-L1 axis to suppress the activity of brain-infiltrating PD- $1^{+}$ immune cells, especially PD- $1^{+} \mathrm{T}$ cells as approximately $25 \%$ of total T cells in the brain of TBI mice were PD-1 ${ }^{+}$ (Fig. 5D), and (2) PD-L1 intrinsic signaling to regulate the timing and intensity of astrocyte reaction to TBI (Fig. 6). Therefore, our research suggests that PD-L1 ${ }^{+}$astrocytes function as a gatekeeper to the brain to control TBIrelated neuroimmune and neuroinflammatory responses, thereby opening a novel avenue to study the role of ICPneuroimmune axes in the pathophysiology of TBI and possibly other neurological disorders. Our research also provides insights into the development of ICP regulators for ameliorating post-TBI damage and promoting brain tissue repair.

In response to $\mathrm{TBI}$, the brain orchestrates a complex reaction of neural and non-neural cells that interact over time to clear debris, protect viable cells, preserve function, and maintain homeostasis [73]. Astrocytes, the most abundant cells in the brain, are pivotal responders to TBI and undergo a significant change in their phenotype, gene expression, and function, resulting in the activation and proliferation, known as reactive astrocytes, astrogliosis, or astrocytosis [74]. Reactive astrocytes are heterogeneous and form scar borders to segregate damaged and inflamed tissue from adjacent neural tissue $[73,75]$. The most scar-forming reactive astrocytes are newly proliferated and characterized by having a large cytoplasmic mass, long branching processes, and high-level expression of intermediate filament proteins, such as GFAP, vimentin, and nestin, allowing these cells to effectively communicate with and affect surrounding neural cells, non-neural cells, and neural vasculature [76]. Our studies for the first time demonstrated that reactive astrocytes exerted regulatory functions through ICP-neuroimmune axes to control inflammation and immune hyper-responses in addition to being a dense physical barrier around TBI injury. Specifically, de novo expression of PD-L1 was robustly and specifically induced in reactive astrocytes (Figs. 1, 2). These PD- $1^{+}$ astrocytes were highly enriched to form a dense zone around the TBI lesion (Fig. 3). Abrogation of PD-L1 signaling enlarged brain tissue cavity size and worsened TBI outcomes, such as motor function and anxiety in mice (Fig. 4). PD-L1 ${ }^{+}$astrocytes can not only suppress the activity of brain-infiltrating PD- ${ }^{+}$immune cells via the PD-1/PD-L1 axis, but also exert PD-L1 intrinsic signaling to down-regulate MCP-1 (CCL2) production (Figs. 5, 6), leading to reduction of inflammatory Ly- $6 C^{\text {High }} M / M \phi$ recruitment. These results strongly imply that $\mathrm{PD}-\mathrm{L} 1^{+}$ astrocytes add a new layer of immune regulation and protection through dual inhibitory functions of PD-L1, providing insights into the mechanisms by which ablation of reactive astrocytes prolongs neuroinflammatory responses and intensifies the damage of TBI [77].

Tissue damage and cellular destruction are the major events in TBI primary injury, which rapidly releases molecules of damage-associated molecular patterns (DAMPs), such as high-mobility group box protein 1 (HMGB1) [78-80]. These DAMPs bind to Toll-like receptors (TLRs) and/or the receptor for advanced glycation end products (RAGE) in neural cells, such as astrocytes, microglia cells, and neurons to trigger activation of nuclear factor kappa B (NF- $\kappa$ B) [78-80], leading to production of inflammatory cytokines and chemokines $[7$, 81-88]. In fact, reactive astrocytes produce a wide range of inflammatory cytokines and chemokines including IFN- $\gamma$, IL- $1 \beta$, TNF- $\alpha$, TGF- $\beta$, IL-6, MCP-1 (CCL2), and CXCL10 [89]. It is well established that these cytokines and chemokines are potent mediators of inflammation and inflammatory cell recruitment and play a detrimental role in secondary injury of TBI [74]. We found that CSF levels of MCP-1 (CCL2) were highly elevated in TBI mice when compared to sham mice and MCP-1 was induced in human U87MG astrocytes in response to stimulation with the proinflammatory cytokines (IFN- $\gamma$, IL-1 $\beta$, and TNF- $\alpha$ ) (Fig. 6). Abrogation of PD-L1 gene in vitro dramatically increased MCP-1 production, indicating that PD-L1 in astrocytes exhibited its inhibitory intrinsic signaling to suppress MCP-1 expression. MCP-1 interacts with its cognate receptor CCR2 that is highly expressed on inflammatory Ly- $6 \mathrm{C}^{\text {High }} \mathrm{M} / \mathrm{M} \phi$ to chemotactically recruit these cells into inflammatory sites $[54,55]$. We found that inflammatory Ly- $6 \mathrm{C}^{\text {High }} \mathrm{M} / \mathrm{M} \phi$ were accumulated in the brain of TBI mice and further increased in the brain of TBI mice after PD-L1 blockade. Notably, tissue-repairing $\mathrm{Ly}-6 \mathrm{C}^{\mathrm{Low}} \mathrm{F} 40 / 80^{+} \mathrm{M} / \mathrm{M} \phi$ that express the chemokine receptor CX3CR1 [90], but no CCR2, in the brain of TBI mice were not affected by PD-L1 blockade (Fig. 5). Based on our findings, we proposed a working model that PD-L1-expressing astrocytes act as a gate-keeper for neuroinflammation in the CNS of TBI mice. TBI-induced DAMPs and inflammatory cytokines activate astrocytes. Some cytokines such as IFN- $\gamma$ can activate astrocytes and are also produced by reactive astrocytes [91], establishing IFN- $\gamma$ autocrine signaling loops to amplify astrocyte activation. In addition, infiltrated immune cells such as $T$ cells, B cells, and $M / M \phi$ produce IFN- $\gamma$, which likely accelerates IFN- $\gamma$ autocrine signals in reactive astrocytes. IFN- $\gamma$ binds to the specific IFN- $\gamma$ receptors 1 and 2 (IFNGR1 and IFNGR2) to activate and recruit Janus kinases 1 and 2 (JAK1 and JAK 2) that serve as a docking site for the signaling transducer and activator of transcription 1 (STAT 1). STAT1 undergoes phosphorylation and homodimerization, and 
dimerized STAT1 translocates to the nucleus and subsequently binds to gamma-activated sequence (GAS) elements in the promotors of IFN- $\gamma$-regulated genes to regulate gene expression, inflammation, and cell-mediated immune responses [92]. Here, we showed that IFN- $\gamma$ rapidly and potently upregulated expression of the inhibitory ICP PD-L1 in astrocytes and that PD-L1 deficiency enhanced MCP-1 production in astrocytes. Our results suggest that the PD-L1 intrinsic signaling acts as a brake on the activity of the IFN- $\gamma /$ JAK/STAT loop in reactive astrocytes, thereby playing a key role in regulation of the timing and intensity of astrocyte reaction to TBI and in prevention of excessive neuroimmune and neuroinflammatory responses to TBI.

ICP blockade has become a revolutionary treatment for several advanced malignancies, such as melanoma and lung cancer $[15,20,31]$. Such success has led to the investigation of ICP blockade therapies for brain metastasis of these malignancies. However, emerging evidence has demonstrated that cancer patients receiving ICP blockade therapies are at risk for developing immune-related neurological complications, such as cranial polyneuropathy and autoimmune encephalitis [15, 93-95]. Although these neurological complications are not very common, their incidence will likely increase as the use of ICP blockade therapies is rapidly growing. Currently, there is a lack of knowledge about ICP axes in the brain and a lack of biomarkers to predict the occurrence and severity of neurological side effects of ICP blockade therapies. Our research demonstrated that PD-L1 was remarkably induced and exhibited biological functions in the brain of TBI mice, providing novel insights into the molecular mechanisms underlying immune regulation in neurological disorders.

\section{Conclusion}

To date, more than 20 ICPs including both inhibitory and stimulatory molecules have been extensively studied in the peripheral circulation but remain elusive in the CNS. These ICPs display great diversity in their expression, regulation, and function, which is largely context or disease dependent. Thus, it is important to understand the role of ICP-neuroimmune axes in the CNS in health and disease, which will reveal important insights into the pathogenesis and treatment of CNS diseases, such as TBI.

\footnotetext{
Abbreviations

TBI: Traumatic brain injury; BBB: Blood-brain barrier; M/M $\phi$ : Monocyte/macrophage; CNS: Central nervous system; ICP: Immune checkpoint; CTLA-4: Cytotoxic T-lymphocyte-associated protein 4; PD-1: Programmed cell death protein 1; PD-L1: Programmed death-ligand 1; TIM-3: T-cell immunoglobulin mucin protein 3; LAG-3: Lymphocyte-activation gene 3; CTL: Cytotoxic T lymphocyte; CCl: Controlled cortical impact; GFAP: Glial fibrillary acidic protein; IACUC: Institutional Animal Care and Use Committee; IP: Intraperitoneal;
}

Ab: Antibody; CSF: Cerebrospinal fluid; PBS: Phosphate-buffered saline; IHC: Immunohistochemistry; PFA: Paraformaldehyde; Iba1: Ionized calcium binding adaptor molecule 1; NeuN: Neuronal nuclei; NG2: Nerve/glial antigen 2; HBSS: Hanks'Balanced Salt Solution; FBS: Fetal bovine serum; RBC: Red blood cell; FVD: Fixable viability dye; PMN: Polymorphonuclear neutrophil; PVDF: Polyvinylidene difluoride; ELISA: Enzyme-linked immunosorbent assay; B7-H1: B7 homolog 1; gRNA: Guide RNA; WT: Wild type; KO: Knockout; EPM: Elevated plus maze; GLMM: Generalized linear mixture model; OPC: Oligodendrocyte progenitor cell.

\section{Supplementary Information}

The online version contains supplementary material available at https://doi. org/10.1186/s12974-022-02398-x.

Additional file 1: Figure S1. PD-L1 Ab via subcutaneous injection reached the injured site and bound to GFAP ${ }^{+}$reactive astrocytes in the brain of TBI mice. Mice $24 \mathrm{~h}$ post-TBI were given PD-L1-Alex647conjugated $\mathrm{Ab}$ (red) or IgG via SC injection. Brain tissues were harvested $48 \mathrm{~h}$ post-injection for IHC analysis of PD-L1 binding. Left panels, the high power images showing that there is no red signal (PD- L1) detected in the brain of TBI mice brain with IgG treatment. Right panels, the high power images showing that PD-L1-Alex647-conjugated Ab (red) reached the injured site and bound to GFAP ${ }^{+}$reactive astrocytes. Similar results were obtained from 3 mice of each group. Figure S2. No significant change of GFAP expression with PD-L1 Ab treatment post-TBI. One week post-TBI (6 days after PD-L1 Ab or lgG treatment), GFAP expression in injured cortex was examined using IF staining. A) and B), GFAP-positive reactive astrocytes (green signal) in injured cortex at 1 week post-TBI with IgG control or PD-L1 Ab treatment. There was no significant difference of GFAP signal between groups. A1) and B1), High power images from the white box of A) and B) showed that PD-L1 Ab treatment had no effect on the morphology of GFAP-positive reactive astrocytes. C), GFAP intensity quantification confirmed that PD-L1 Ab treatment did not change the GFAP expression after TBI. ns, not significant.

\section{Acknowledgements}

We would especially like to thank Dr. Jinhui Chen (chenjh89@gmail.com) for his support and contributions to the conception, experimental design, data gathering, and discussion when he was in Indiana University School of Medicine.

\section{Author contributions}

All authors conceived of and helped direct the study. XG, WL, FS, PL, and FY performed the experiments and analyses. All authors contributed to writing this paper. All authors read and approved the final manuscript.

\section{Funding}

This work was supported in part by NIH UH2/UH3 AA026218 (QY), R21/R33 Al104268 (QY), R01 Al1 17835 (QY), the Grand Challenges Explorations (GCE) Phase II grant through the Bill \& Melinda Gates Foundation (OPP1035237 to QY), the Showalter Research Trust Fund (QY), Indiana Spinal Cord \& Brain Injury Research Fund (ISCBIRF) from the Indiana State Department of Health (XG), and the Research Facilities Improvement Program Grant Number C06 RR015481-01 from the National Center for Research Resources, NIH, to Indiana University School of Medicine.

\section{Availability of data and materials}

The data used in this study are available from the corresponding authors up on reasonable request.

\section{Declarations}

Ethics approval and consent to participate Not applicable.

\section{Consent for publication}

Not applicable. 


\section{Competing interest}

The authors declare that they have no competing interests.

\section{Author details}

${ }^{1}$ Spinal Cord and Brain Injury Research Group, Department of Neurological Surgery, Stark Neurosciences Research Institute, Indiana University School of Medicine, 320 W. 15th Street, Indianapolis, IN 46202, USA. ${ }^{2}$ Department of Microbiology and Immunology, Medical Science Building, MS267, Indiana University School of Medicine, 635 Barnhill Drive, Indianapolis, IN 46202, USA ${ }^{3}$ Department of Surgery, Indiana University School of Medicine, Indianapolis, IN 46202, USA

\section{Received: 12 August 2021 Accepted: 24 January 2022}

\section{Published online: 08 February 2022}

\section{References}

1. Kolias AG, Rubiano AM, Figaji A, Servadei F, Hutchinson PJ. Traumatic brain injury: global collaboration for a global challenge. Lancet Neurol. 2019;18:136-7.

2. Johnson WD, Griswold DP. Traumatic brain injury: a global challenge. Lancet Neurol. 2017;16:949-50.

3. Hyder AA, Wunderlich CA, Puvanachandra P, Gururaj G, Kobusingye OC The impact of traumatic brain injuries: a global perspective. NeuroRehabilitation. 2007;22:341-53.

4. Nasr IW, Chun Y, Kannan S. Neuroimmune responses in the developing brain following traumatic brain injury. Exp Neurol. 2019:320:112957.

5. Jassam YN, Izzy S, Whalen M, McGavern DB, El Khoury J. Neuroimmunology of traumatic brain injury: time for a paradigm shift. Neuron. 2017:95:1246-65

6. McKee AC, Daneshvar DH. The neuropathology of traumatic brain injury Handb Clin Neurol. 2015:127:45-66.

7. Corps KN, Roth TL, McGavern DB. Inflammation and neuroprotection in traumatic brain injury. JAMA Neurol. 2015;72:355-62.

8. Morganti-Kossmann MC, Satgunaseelan L, Bye N, Kossmann T. Modulation of immune response by head injury. Injury. 2007;38:1392-400.

9. Xiong Y, Mahmood A, Chopp M. Current understanding of neuroinflammation after traumatic brain injury and cell-based therapeutic opportunities. Chin J Traumatol. 2018:21:137-51.

10. Kumar A, Loane DJ. Neuroinflammation after traumatic brain injury: opportunities for therapeutic intervention. Brain Behav Immun. 2012;26:1191-201.

11. Kumar A, et al. Microglial-derived microparticles mediate neuroinflammation after traumatic brain injury. J Neuroinflamm. 2017;14:47.

12. Davalos $D$, et al. ATP mediates rapid microglial response to local brain injury in vivo. Nat Neurosci. 2005;8:752-8.

13. Block ML, Zecca L, Hong JS. Microglia-mediated neurotoxicity: uncovering the molecular mechanisms. Nat Rev Neurosci. 2007;8:57-69.

14. Zhang D, Hu X, Qian L, O'Callaghan JP, Hong JS. Astrogliosis in CNS pathologies: is there a role for microglia? Mol Neurobiol. 2010;41:232-41.

15. Berghoff AS, Venur VA, Preusser M, Ahluwalia MS. Immune checkpoint inhibitors in brain metastases: from biology to treatment. Am Soc Clin Oncol Educ Book. 2016;35:e116-122.

16. Galea I, Bechmann I, Perry VH. What is immune privilege (not)? Trends Immunol. 2007;28:12-8.

17. Sanmamed MF, Chen L. A paradigm shift in cancer immunotherapy: from enhancement to normalization. Cell. 2018;175:313-26.

18. Chen L, Flies DB. Molecular mechanisms of T cell co-stimulation and coinhibition. Nat Rev Immunol. 2013;13:227-42.

19. Sharma P, Allison JP. The future of immune checkpoint therapy. Science. 2015;348:56-61.

20. Wei SC, Duffy CR, Allison JP. Fundamental mechanisms of immune checkpoint blockade therapy. Cancer Discov. 2018;8:1069-86.

21. Ishida Y, Agata Y, Shibahara K, Honjo T. Induced expression of PD-1, a novel member of the immunoglobulin gene superfamily, upon programmed cell death. EMBO J. 1992;11:3887-95

22. Wykes MN, Lewin SR. Immune checkpoint blockade in infectious diseases. Nat Rev Immunol. 2018;18:91-104.

23. Dyck L, Mills KHG. Immune checkpoints and their inhibition in cancer and infectious diseases. Eur J Immunol. 2017;47:765-79.
24. Naran K, Nundalall T, Chetty S, Barth S. Principles of immunotherapy: implications for treatment strategies in cancer and infectious diseases. Front Microbiol. 2018:9:3158.

25. Rao M, Valentini D, Dodoo E, Zumla A, Maeurer M. Anti-PD-1/PD-L1 therapy for infectious diseases: learning from the cancer paradigm. Int $J$ Infect Dis. 2017;56:221-8.

26. Trautmann $L$, et al. Upregulation of PD-1 expression on HIV-specific CD8 + T cells leads to reversible immune dysfunction. Nat Med. 2006:12:1198-202.

27. Kaufmann DE, et al. Upregulation of CTLA-4 by HIV-specific CD4+T cells correlates with disease progression and defines a reversible immune dysfunction. Nat Immunol. 2007;8:1246-54.

28. Chew GM, et al. TIGIT marks exhausted t cells, correlates with disease progression, and serves as a target for immune restoration in HIV and SIV infection. PLoS Pathog. 2016;12:e1005349.

29. Topalian SL, Drake CG, Pardoll DM. Immune checkpoint blockade: a common denominator approach to cancer therapy. Cancer Cell. 2015:27:450-61.

30. Pardoll DM. The blockade of immune checkpoints in cancer immunotherapy. Nat Rev Cancer. 2012;12:252-64.

31. Hargadon KM, Johnson CE, Williams CJ. Immune checkpoint blockade therapy for cancer: an overview of FDA-approved immune checkpoint inhibitors. Int Immunopharmacol. 2018;62:29-39.

32. Frost RB, Farrer TJ, Primosch $M$, Hedges DW. Prevalence of traumatic brain injury in the general adult population: a meta-analysis. Neuroepidemiology. 2013;40:154-9.

33. Lagbas C, Bazargan-Hejazi S, Shaheen M, Kermah D, Pan D. Traumatic brain injury related hospitalization and mortality in California. Biomed Res Int. 2013:2013:143092.

34. Gao $X$, Wang $X$, Xiong $W$, Chen J. In vivo reprogramming reactive glia into iPSCs to produce new neurons in the cortex following traumatic brain injury. Sci Rep. 2016;6:22490.

35. Zhao S, Gao X, Dong W, Chen J. The role of 7,8-dihydroxyflavone in preventing dendrite degeneration in cortex after moderate traumatic brain injury. Mol Neurobiol. 2016;53:1884-95.

36. Gao X, Deng P, Xu ZC, Chen J. Moderate traumatic brain injury causes acute dendritic and synaptic degeneration in the hippocampal dentate gyrus. PLoS One. 2011;6:e24566.

37. Han R, Luo J, Shi Y, Yao Y, Hao J. PD-L1 (Programmed Death Ligand 1) protects against experimental intracerebral hemorrhage-induced brain injury. Stroke. 2017:48:2255-62.

38. Bodhankar S, et al. PD-L1 monoclonal antibody treats ischemic stroke by controlling central nervous system inflammation. Stroke. 2015:46:2926-34.

39. Gao X, Chen J. Moderate traumatic brain injury promotes neural precursor proliferation without increasing neurogenesis in the adult hippocampus. Exp Neurol. 2013;239:38-48.

40. Smith $\mathrm{DH}$, et al. A model of parasagittal controlled cortical impact in the mouse: cognitive and histopathologic effects. J Neurotrauma. 1995;12:169-78.

41. Hamm RJ, Pike BR, O'Dell DM, Lyeth BG, Jenkins LW. The rotarod test: an evaluation of its effectiveness in assessing motor deficits following traumatic brain injury. J Neurotrauma. 1994:11:187-96.

42. Rochat $L$, et al. Assessment of impulsivity after moderate to severe traumatic brain injury. Neuropsychol Rehabil. 2010;20:778-97.

43. Wu D, Qiu Y, Gao X, Yuan XB, Zhai Q. Overexpression of SIRT1 in mouse forebrain impairs lipid/glucose metabolism and motor function. PLoS One. 2011:6:e21759.

44. Popovitz J, Mysore SP, Adwanikar H. Long-term effects of traumatic brain injury on anxiety-like behaviors in mice: behavioral and neural correlates. Front Behav Neurosci. 2019;13:6

45. Lim NK, et al. An improved method for collection of cerebrospinal fluid from anesthetized mice. J Vis Exp. 2018. https://doi.org/10.3791/56774.

46. Glosson-Byers NL, et al. Th17 cells demonstrate stable cytokine production in a proallergic environment. J Immunol. 2014;193:2631-40.

47. Fabrizio FP, et al. Gene code CD274/PD-L1: from molecular basis toward cancer immunotherapy. Ther Adv Med Oncol. 2018;10:1758835918815598. 
48. Mitchell AL, et al. Programmed death ligand 1 (PD-L1) gene variants contribute to autoimmune Addison's disease and Graves' disease susceptibility. J Clin Endocrinol Metab. 2009;94:5139-45.

49. Mahoney KM, et al. A secreted PD-L1 splice variant that covalently dimerizes and mediates immunosuppression. Cancer Immunol Immunother. 2019;68:421-32

50. Meier JA, Zhang F, Sanjana NE. GUIDES: sgRNA design for loss-of-function screens. Nat Methods. 2017;14:831-2.

51. Ran FA, et al. Genome engineering using the CRISPR-Cas9 system. Nat Protoc. 2013;8:2281-308.

52. Lagos-Cabre R, Burgos-Bravo F, Avalos AM, Leyton L. Connexins in astrocyte migration. Front Pharmacol. 2019;10:1546.

53. Zhao S, Wang X, Gao X, Chen J. Delayed and progressive damages to juvenile mice after moderate traumatic brain injury. Sci Rep. 2018;8:7339.

54. Jia T, et al. Additive roles for MCP-1 and MCP-3 in CCR2-mediated recruitment of inflammatory monocytes during Listeria monocytogenes infection. J Immunol. 2008;180:6846-53.

55. Hammond MD, et al. CCR2+ Ly6C(hi) inflammatory monocyte recruitment exacerbates acute disability following intracerebral hemorrhage. J Neurosci. 2014;34:3901-9.

56. Bengsch B, et al. Bioenergetic insufficiencies due to metabolic alterations regulated by the inhibitory receptor PD-1 are an early driver of CD8(+) T cell exhaustion. Immunity. 2016;45:358-73.

57. Patsoukis N, et al. PD-1 alters T-cell metabolic reprogramming by inhibiting glycolysis and promoting lipolysis and fatty acid oxidation. Nat Commun. 2015;6:6692

58. Freeman GJ, et al. Engagement of the PD-1 immunoinhibitory receptor by a novel B7 family member leads to negative regulation of lymphocyte activation. J Exp Med. 2000;192:1027-34.

59. Keir ME, et al. Tissue expression of PD-L1 mediates peripheral T cell tolerance. J Exp Med. 2006:203:883-95.

60. Dong $\mathrm{H}$, et al. Tumor-associated B7-H1 promotes T-cell apoptosis: a potential mechanism of immune evasion. Nat Med. 2002;8:793-800.

61. Keir ME, Butte MJ, Freeman GJ, Sharpe AH. PD-1 and its ligands in tolerance and immunity. Annu Rev Immunol. 2008;26:677-704.

62. Dong H, Zhu G, Tamada K, Chen L. B7-H1, a third member of the B7 family, co-stimulates T-cell proliferation and interleukin-10 secretion. Nat Med. 1999:5:1365-9.

63. Chemnitz JM, Parry RV, Nichols KE, June CH, Riley JL. SHP-1 and SHP-2 associate with immunoreceptor tyrosine-based switch motif of programmed death 1 upon primary human T cell stimulation, but only receptor ligation prevents T cell activation. J Immunol. 2004;173:945-54.

64. Yokosuka T, et al. Programmed cell death 1 forms negative costimulatory microclusters that directly inhibit T cell receptor signaling by recruiting phosphatase SHP2. J Exp Med. 2012;209:1201-17.

65. Gato-Canas M, et al. PDL1 signals through conserved sequence motifs to overcome interferon-mediated cytotoxicity. Cell Rep. 2017;20:1818-29.

66. Dong P, Xiong Y, Yue J, Hanley SJB, Watari H. Tumor-ilntrinsiC PD-L1 signaling in cancer initiation, development and treatment: beyond immune evasion. Front Oncol. 2018;8:386.

67. Poggio M, et al. Suppression of exosomal PD-L1 induces systemic antitumor immunity and memory. Cell. 2019;177:414-427.e413.

68. Hartley GP, Chow L, Ammons DT, Wheat WH, Dow SW. Programmed Cell Death Ligand 1 (PD-L1) signaling regulates macrophage proliferation and activation. Cancer Immunol Res. 2018:6:1260-73.

69. Jalali S, et al. Reverse signaling via PD-L1 supports malignant cell growth and survival in classical Hodgkin lymphoma. Blood Cancer J. 2019;9:22.

70. Lecis D, Sangaletti S, Colombo MP, Chiodoni C. Immune checkpoint ligand reverse signaling: looking back to go forward in cancer therapy. Cancers (Basel). 2019;11:624.

71. Lomas-Neira J, et al. Novel role for PD-1:PD-L1 as mediator of pulmonary vascular endothelial cell functions in pathogenesis of indirect ARDS in mice. Front Immunol. 2018;9:3030.

72. Butte MJ, Keir ME, Phamduy TB, Sharpe AH, Freeman GJ. Programmed death-1 ligand 1 interacts specifically with the B7-1 costimulatory molecule to inhibit T cell responses. Immunity. 2007;27:111-22.

73. Burda JE, Sofroniew MV. Reactive gliosis and the multicellular response to CNS damage and disease. Neuron. 2014;81:229-48.

74. Sofroniew MV. Molecular dissection of reactive astrogliosis and glial scar formation. Trends Neurosci. 2009;32:638-47.
75. Sofroniew MV, Vinters HV. Astrocytes: biology and pathology. Acta Neuropathol. 2010;119:7-35.

76. Wanner IB, et al. Glial scar borders are formed by newly proliferated, elongated astrocytes that interact to corral inflammatory and fibrotic cells via STAT3-dependent mechanisms after spinal cord injury. J Neurosci. 2013;33:12870-86.

77. Burda JE, Bernstein AM, Sofroniew MV. Astrocyte roles in traumatic brain injury. Exp Neurol. 2016;275(Pt 3):305-15.

78. Piccinini AM, Midwood KS. DAMPening inflammation by modulating TLR signalling. Mediators Inflamm. 2010;2010:1.

79. Thakur KK, et al. Therapeutic implications of toll-like receptors in peripheral neuropathic pain. Pharmacol Res. 2017;115:224-32.

80. Anders HJ, Schaefer L. Beyond tissue injury-damage-associated molecular patterns, toll-like receptors, and inflammasomes also drive regeneration and fibrosis. J Am Soc Nephrol. 2014;25:1387-400.

81. Farina C, Aloisi F, Meinl E. Astrocytes are active players in cerebral innate immunity. Trends Immunol. 2007;28:138-45.

82. Lehnardt S. Innate immunity and neuroinflammation in the CNS: the role of microglia in Toll-like receptor-mediated neuronal injury. Glia. 2010;58:253-63.

83. Rivest $\mathrm{S}$. Regulation of innate immune responses in the brain. Nat Rev Immunol. 2009;9:429-39.

84. Laird MD, et al. High mobility group box protein-1 promotes cerebral edema after traumatic brain injury via activation of toll-like receptor 4 Glia. 2014;62:26-38.

85. Wang KY, Yu GF, Zhang ZY, Huang Q, Dong XQ. Plasma high-mobility group box 1 levels and prediction of outcome in patients with traumatic brain injury. Clin Chim Acta. 2012;413:1737-41.

86. Au AK, et al. Cerebrospinal fluid levels of high-mobility group box 1 and cytochrome C predict outcome after pediatric traumatic brain injury. J Neurotrauma. 2012;29:2013-21.

87. Hayakawa K, Pham LD, Katusic ZS, Arai K, Lo EH. Astrocytic high-mobility group box 1 promotes endothelial progenitor cell-mediated neurovascular remodeling during stroke recovery. Proc Natl Acad Sci U S A. 2012:109:7505-10.

88. Hayakawa K, et al. High-mobility group box 1 from reactive astrocytes enhances the accumulation of endothelial progenitor cells in damaged white matter. J Neurochem. 2013;125:273-80.

89. Nutma E, van Gent D, Amor S, Peferoen LAN. Astrocyte and oligodendrocyte cross-talk in the central nervous system. Cells. 2020;9:600.

90. Olingy $C E$, et al. Non-classical monocytes are biased progenitors of wound healing macrophages during soft tissue injury. Sci Rep. 2017;7:447.

91. Hashioka S, et al. Interferon-gamma-induced neurotoxicity of human astrocytes. CNS Neurol Disord Drug Targets. 2015;14:251-6.

92. Michalska A, Blaszczyk K, Wesoly J, Bluyssen HAR. A positive feedback amplifier circuit that regulates interferon (IFN)-stimulated gene expression and controls type $\mathrm{i}$ and type II IFN responses. Front Immunol. 2018:9:1135.

93. Hottinger AF. Neurologic complications of immune checkpoint inhibitors Curr Opin Neurol. 2016;29:806-12.

94. Fellner A, et al. Neurologic complications of immune checkpoint inhibitors. J Neurooncol. 2018:137:601-9.

95. Dalakas MC. Neurological complications of immune checkpoint inhibitors: what happens when you "take the brakes off" the immune system. Ther Adv Neurol Disord. 2018;11:1756286418799864.

\section{Publisher's Note}

Springer Nature remains neutral with regard to jurisdictional claims in published maps and institutional affiliations. 